\title{
Conectando un territorio: simulación de rutas de movilidad entre cazadores-recolectores y primeros cultivadores. EI caso del Cauca medio (Macizo Volcánico, Colombia)
}

\author{
Connecting a territory: modelling routes of mobility between hunter-gatherers and early \\ plant cultivators. The case of the Middle Cauca (Macizo Volcánico, Colombia)
}

Francisco Javier Aceituno Bocanegra ${ }^{a}$ y Antonio Uriarte González ${ }^{b}$

RESUMEN

La movilidad es una de las principales características de los grupos cazadores-recolectores e incipientes cultivadores. A partir de los años 90 y a nivel global, los estudios sobre movilidad experimentan un fuerte avance gracias a la aplicación de los Sistemas de Información Geográfica (SIG), con los análisis de los costes de desplazamiento y el modelado de rutas, así como la relación de estos elementos con el acceso a los recursos, la interacción entre grupos y la territorialidad. El presente artículo se basa en la simulación de rutas de movilidad en el Cauca medio, región andina localizada en el Macizo Volcánico colombiano (Cordillera Central), donde se cuenta con una secuencia muy completa de ocupaciones precerámicas entre el Pleistoceno final y el Holoceno medio. Esta tarea se ha abordado, en el marco de los SIG, mediante herramientas de análisis de costes, concretamente el análisis Modelo de Acumulación del Desplazamiento Óptimo desde un origen (MADO) y la generación de caminos óptimos. Los resultados muestran una asociación significativa entre yacimientos arqueológicos y rutas potenciales, así como una red de movilidad coherente y articulada.

\begin{abstract}
Mobility is one of the main characteristics of hunter-gatherers and early plant cultivators. Since the 90s, world-wide, mobility studies have made strong progress thanks to the application of Geographic Information Systems (GIS), involving travel-cost analysis and route modelling, and how these relate to resource accessibility, group interaction, and territoriality. This paper presents a simulation of mobility routes in the Middle Cauca, an Andean
\end{abstract}

region located in the Colombian Macizo Volcánico (Cordillera Central), where there is a very complete sequence of preceramic occupations from the Late Pleistocene to the Middle Holocene. We use GIS to tackle this task, by means of cost analysis tools, specifically MADO analysis and least-cost paths generation. Results show a significant association between archaeological sites and potential routes, as well as a coherent and articulated mobility network.

Palabras clave: Movilidad; Sistemas de Información Geográfica; Análisis de costes; Modelo de Acumulación del Desplazamiento Óptimo (MADO); Caminos óptimos; Colombia; Cauca medio; Cazadores-recolectores; Primeros cultivadores; Pleistoceno final; Holoceno temprano y medio.

Key words: Mobility; Geographic Information Systems; Cost analysis; MADO; Least-cost path; Colombia; Middle Cauca; Hunter-gatherers; Early cultivators; Late Pleistocene; Early and Middle Holocene.

\section{INTRODUCCIÓN}

En sociedades nómadas, la movilidad en su acepción más básica es una estrategia adaptativa para hacer frente a la diversidad medioambiental y a la distribución temporal y espacial de los recursos (Kelly 1995; Lee y Daly 1999: 4). En la arqueología de cazadores-recolectores, el enfoque neofuncionalista de los trabajos de Binford (1979, 1980, 1987, 2001) marcó un punto de inflexión en los estudios de movilidad. Binford definió dos modelos para enfrentar

\footnotetext{
a Grupo Medio Ambiente y Sociedad, Departamento de Antropología. Universidad de Antioquia. Calle 67 n. ${ }^{\circ} 53-108$. AA 1226 Antioquia. Colombia. Correo e.: francisco.aceituno@udea.edu.co https://orcid.org/0000-0002-5727-8826

b Laboratorio de Arqueología del Paisaje y Teledetección, Instituto de Historia. Consejo Superior de Investigaciones Científicas. C/ Albasanz 2628. 28037 Madrid. España. Correo e.: antonio.uriarte@cchs.csic.es https://orcid.org/0000-0001-9165-957X

Recibido 05-VII-2018; aceptado 24-IV-2019.
} 
la variabilidad espacial y temporal de los recursos (Fig. 1). El "modelo forrajero" comprende los movimientos de un grupo de un campamento a otro, hacia donde se localizan los recursos (movilidad residencial). El "modelo colector" consiste en desplazamientos de unos pocos individuos para realizar actividades concretas en lugares específicos y el regreso a los campamentos residenciales (movilidad logística) (Kelly 1995: 117).

La "movilidad residencial" se asoció con pequeños grupos viviendo en biomas sin estaciones térmicas marcadas y con ambientes donde los recursos se distribuyen, principalmente, de forma dispersa a lo largo de todo el año, como es el caso de los trópicos. En contraste, la "movilidad logística" es propia de ambientes con estacionalidad térmica marcada y con una distribución concentrada de los recursos en el tiempo y el espacio, como son los biomas de las zonas templadas (Binford 1980, 1990). Binford trató de definir el correlato arqueológico de ambos modelos, apoyándose en referentes etnoarqueológicos. Como resultado, en los años 80 y 90, los estudios de movilidad estuvieron muy marcados por la relación entre movilidad y tecnología lítica. Tipos de asentamiento y modelos de movilidad se analizaron a partir del diseño de los artefactos líticos y el patrón de descarte de los sitios arqueológicos (Binford 1979, 1980; Torrence 1983, 1989; Shott 1986, 1996; Lurie 1989; Bettinger 1991: 69; Nelson 1991; Kelly 1992; Cowan 1999; Shoocongdej 2000; Odell 2004: 10).

Los enfoques anteriores se basaron sobre todo en la tecnología lítica, en el patrón de descarte y en la explotación de recursos bióticos y obtención de materias primas, desde modelos ecológicos basados princi-

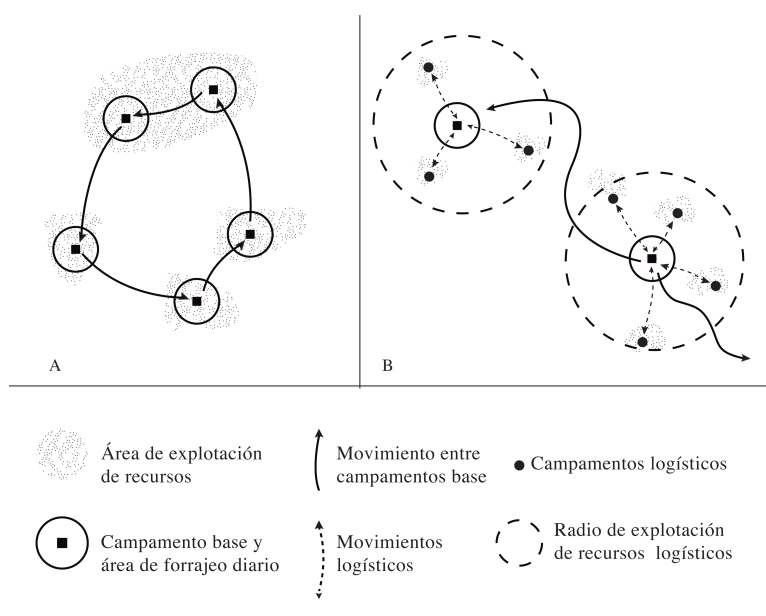

Fig. 1. Modelos de movilidad: A. M. residencial ("modelo forrajero"). B. M. logística ("modelo colector"). Figura adaptada de P. Rowley-Conwy (2001: 41) basada en la discusión de Binford (1980). palmente en la relación coste-beneficio. El análisis de rutas se centró en la relación entre localización de fuentes de materia prima y movilidad (Aubry et al. 2012; Li et al. 2016), dado que los recursos líticos son menos susceptibles a cambios que, por ejemplo, los recursos bióticos. Los estudios sobre movilidad, incluyendo las rutas, reciben un fuerte impulso con la aplicación de los Sistemas de Información Geográfica (SIG) a la arqueología y el inicio de los análisis a escala macro-espacial, que, en términos generales, han permitido estudiar las formas de movimiento sobre el territorio y entender mejor el rol de la movilidad como estrategia de uso del espacio, interacción y comunicación entre poblaciones humanas. Los SIG han permitido interpretar el registro arqueológico en clave de movilidad y profundizar en el análisis de las redes de intercambio y territorialidad en sociedades cazadorasrecolectoras (Brantingham 2006; Aubry et al. 2012; Sauvet 2017) (véanse desarrollo y referencias en el apartado 3).

El estudio de la movilidad mediante análisis SIG ha sido aplicado, a diferentes escalas y con enfoques y objetivos diversos, en algunos contextos de cazadores-recolectores del continente americano (Morgan 2008, 2009; Magnin et al. 2012; Bruggencate et al. 2016; Cortegoso et al. 2016; Aceituno y Uriarte 2018) y de otras regiones del planeta (Field et al. 2007; Aguilella 2011; Aubry et al. 2012; Byrd et al. 2016), aunque dichos trabajos aún son escasos y dispersos. El presente artículo, al sumarse a esta línea de investigación, pretende contribuir al conocimiento global de las pautas de movilidad en sociedades de pequeña escala y al desarrollo de metodologías que permitan profundizar en este tema.

Además del acceso a los recursos, la movilidad es una acción o estrategia que forma parte de la conducta territorial de los grupos humanos, encaminada a ocupar y controlar un territorio, así como a legitimar su uso para las generaciones venideras. Una de las razones para analizar la movilidad es comprender, aun a nivel básico, el concepto de territorialidad, entendido como el conjunto de estrategias que permiten ejercer algún tipo de control sobre los recursos básicos de una sociedad delimitados espacialmente (Peterson 1975; Dyson-Hudson y Smith 1978; Cashdan 1983; Kelly 1995: 163). Los estudios sobre territorialidad en sociedades cazadoras-recolectoras e incipientes cultivadores coinciden en que los límites territoriales son flexibles, en función de la movilidad (capacidad para moverse), distribución espacial (efecto llamada) y accesibilidad a los recursos. En parte la accesibilidad a nuevas zonas en diferentes escalas espaciales (p. ej. local o regional) depende de las redes de intercambio y de los tipos de relaciones con otros grupos (Sauvet 2017). 
En este escenario de espacialidades, las redes de caminos fijan la movilidad en el territorio. Los caminos conectan y articulan diferentes escalas espaciales, posibilitando redes de intercambio, que se basan en las necesidades de interacción entre grupos y que permiten compartir recursos materiales, información estratégica y bienes culturales con una fuerte carga simbólica; además, pueden ayudar a evitar conflictos y fricciones (Sauvet 2017). En suma, las rutas son constructos que conectan los espacios de la arquitec- tura del territorio, sobre el cual se manifiestan las múltiples formas culturales y sociales de apropiación del espacio (Criado 1999).

Este trabajo busca modelar la movilidad en el Cauca medio, región localizada en los Andes colombianos, en el noroccidente de Suramérica (Fig. 2). A escala macro, este ejercicio analítico aportará información para entender la configuración territorial de los grupos tempranos de esta región, con base en la vinculación de diferentes zonas a través del trazado de potenciales

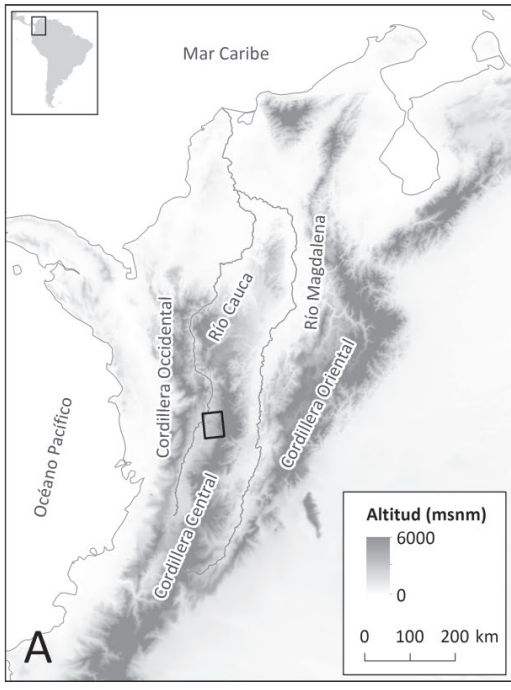

1. La Selva

2. 39 El Recreo Cancha

3. 107 El Perro

4. Los Arrayanes

5. El Antojo

6. Invias 3

7. El Jazmín

8. Guayabito

9. La Pochola

10. San Germán

11. La Romelia

12. La Chillona

13. Campoalegre

14. La Montañita

15. Nuevo Sol

16. Cuba

17. La Trinidad I

18. La Trinidad II

19. El Guatín

20. La Mikela

21. UTP Jardín Botánico

22. Génova

23. Salento 21

24. Salento 24

25. Chaguala

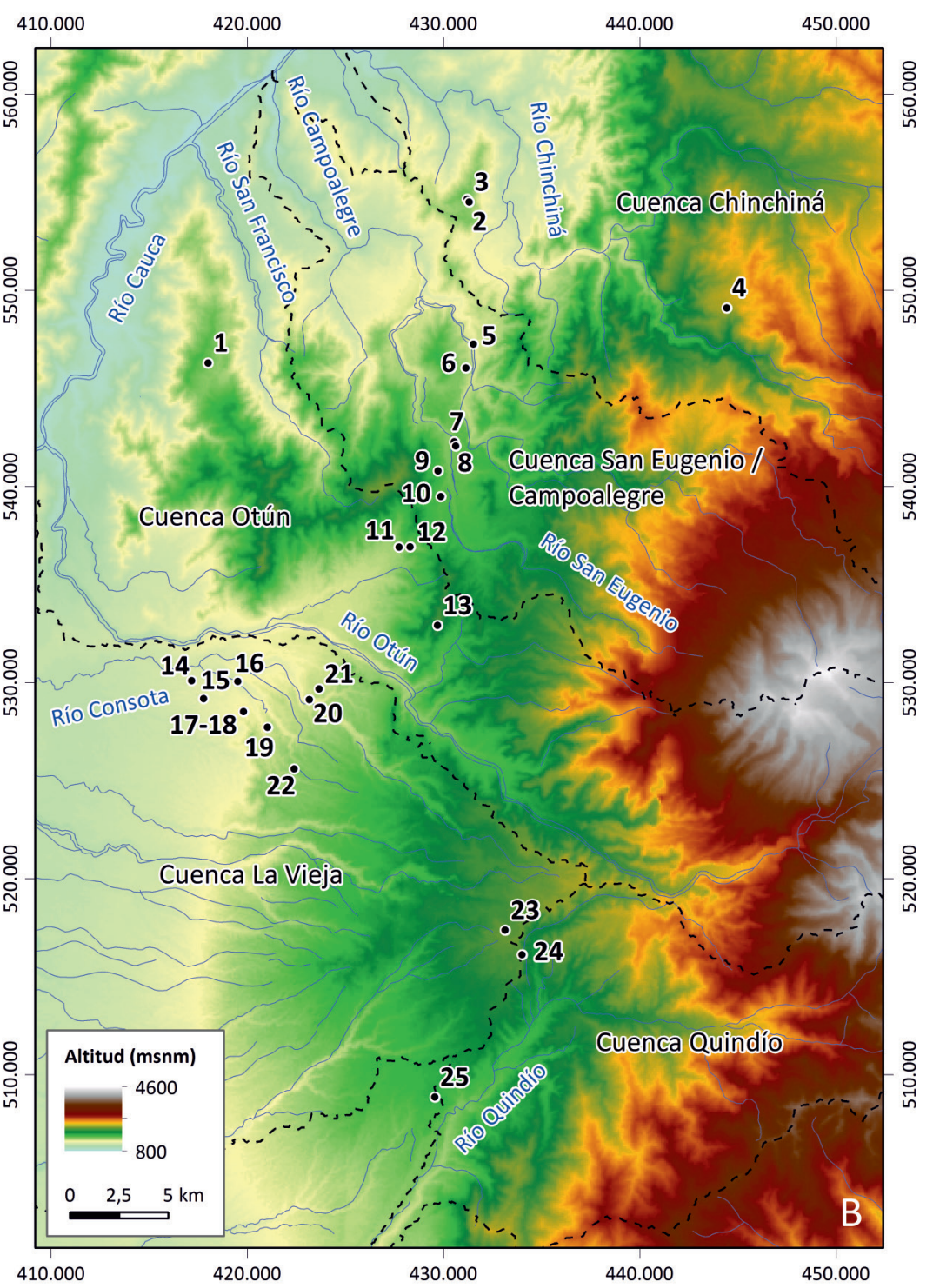

Fig. 2. Localización del Cauca medio (Macizo Volcánico, Colombia). A. Cartografía base: Global 30 Arc-Second Elevation (GTOPO30) (datos disponibles en el U.S. Geological Survey). B. Localización en el Cauca medio de los sitios arqueológicos (véase AC1). Cartografía base: CGIAR-CSI SRTM 90m Digital Elevation Data (Jarvis et al. 2008); Hidrografía de Colombia, Instituto Geográfico Agustín Codazzi (IGAC) (en color en la versión electrónica). 
rutas de comunicación. Tal vinculación únicamente es un primer paso para responder a la pregunta sobre la construcción territorial, cuya respuesta cabal requiere datos de diferente naturaleza. En una escala menor, pretendemos evaluar la relación entre el patrón de distribución y las rutas de tránsito a partir del nexo existente entre las rutas transitables y el patrón espacial del registro arqueológico. Dada la ausencia de vestigios directos de caminos para el período que tratamos aquí, escogimos la modelización como metodología para trazar en el espacio dichas rutas y poner a prueba su rol en la movilidad y en la construcción del espacio.

Nuestros resultados son un primer paso en el estudio de la movilidad en esta región de los Andes colombianos; por lo tanto, temas relacionados como el análisis de las redes de intercambio, escalas y límites territoriales o las formas de apropiación y percepción del territorio, requerirán futuros trabajos en el Cauca medio. En Colombia, el estudio de caminos o rutas de comunicación se ha centrado exclusivamente en sociedades cacicales a partir de dos tipos de fuentes: unas son las referencias sobre intercambios de mercancías en las crónicas de Indias; las segundas son trabajos de campo en la Sierra Nevada de Santa Marta, San Agustín, el valle del Aburrá y el valle del río Calima, donde se han documentado vestigios de caminos que incluyen movimientos de tierra, aterrazamientos o complejos empedrados (Oyuela-Caicedo 1990; Cardale 2000: 45-48; Langebaek 2001; Botero 2007).

\section{CARACTERIZACIÓN GEOGRÁFICA Y ANTECEDENTES ARQUEOLÓGICOS DEL ÁREA DE ESTUDIO}

La región del Cauca medio está ubicada en el Macizo Volcánico colombiano (Cordillera Central). Allí, actualmente, en unos $1.500 \mathrm{~km}^{2}$, se localizan 25 sitios precerámicos (Fig. 2; Anexo AC1) datados entre el Pleistoceno final y el Holoceno medio (Dickau et al. 2015). Sus ocupantes vivieron de la caza, la recolección y el cultivo de plantas (Aceituno y Loaiza 2007, 2014, 2018; Aceituno et al. 2013). La zona de estudio está en la margen derecha del río Cauca, en el piedemonte occidental del Macizo Volcánico, en una franja altitudinal entre los $1.279 \mathrm{~m} \mathrm{~s}$. n. m. del sitio Cuba (16) y los 2.275 m s. n. m. del sitio Los Arrayanes (4) para un promedio de $1.599 \mathrm{~m} \mathrm{~s}$. n. m. Los sitios entre 1.000 y $2.000 \mathrm{~m} \mathrm{~s}$. n. m. pertenecen al piso térmico templado y a la zona de vida bosque muy húmedo montano bajo (Espinal 1985). Los sitios por encima de los $2.000 \mathrm{~m}$ s. n. m. corresponden a la zona de vida bosque muy húmedo premontano, en el piso térmico frío: Los Arrayanes (4), Campoalegre (13), Salento 21 (23) y Salento 24 (24).
Los sitios tienden a concentrarse en dos grandes zonas, al norte y al sur. En la septentrional, los sitios están vinculados principalmente a las cuencas de los ríos Campoalegre y Chinchiná, y en menor medida a los ríos Otún y San Francisco. En la meridional, estos están claramente concentrados en torno al río Consota, que pertenece a la cuenca del río La Vieja. Al sureste de estos sitios, a unos $25 \mathrm{~km}$, se localizan los de Chaguala (25), en la cuenca del río Quindío, y Salento 21 (23) y Salento 24 (24), en el interfluvio entre las cuencas de La Vieja y Quindío (Anexo AC1).

En la zona norte, los sitios están en los valles fluviales, sobre antiguas terrazas que actualmente forman un paisaje de colinas redondeadas con pendientes poco empinadas, erosionadas por cursos de agua y cubiertas por espesas cenizas volcánicas, que es el principal material parental de los profundos suelos de la zona (Thouret 1983). Define la zona sur el abanico fluviovolcánico Pereira-Armenia, una extensa planicie constituida por potentes sedimentos volcánicos que han sido disectados y denudados por los ríos Otún, Consota, La Vieja y Quindío. Sobre los pequeños valles resultantes se hallan los sitios arqueológicos. El análisis del vecino más próximo corrobora la preferencia por un patrón de poblamiento concentrado, en zonas de valle y relieves suaves ${ }^{1}$.

Una característica muy importante de la zona es la naturaleza volcánica de los suelos, pertenecientes al orden andisol. Los andisoles son suelos profundos, con horizontes edafológicos muy bien desarrollados, ricos en materia orgánica y muy fértiles. En este caso las cenizas proceden de volcanes como el Nevado del Ruiz, el Nevado del Tolima, el Santa Isabel y el Cerro Bravo (Cortés 1978).

La presencia de cenizas en los suelos de la zona siempre ha suscitado un gran interés sobre los efectos de la actividad volcánica en las ocupaciones humanas del Cauca medio. La continuidad en el registro arqueológico y las fechas de radiocarbono (Fig. 3; Anexo AC1) (Aceituno y Loaiza 2007; Dickau et al. 2015) indican que los fuertes episodios volcánicos registrados no provocaron el abandono de la región, de lo que cabe deducir que las poblaciones locales se adaptaron de alguna forma a su vulcanismo. Ello no excluye que los grupos sufrieran los efectos perniciosos de las erupciones volcánicas y tuvieran que desplazarse a territorios adyacentes, pero esta hipótesis, hasta la fecha, no ha sido probada aún. La formación de suelos fértiles pudo ser un factor atrayente para las poblaciones humanas a lo largo del Holoceno (Dickau et al. 2015).

\footnotetext{
${ }^{1}$ Análisis del vecino más próximo realizado mediante el paquete ArcToolbox de ArcGIS, con la herramienta Spatial Statistics ToolsAnalyzing Patterns-Average Nearest Neighbor: índice del vecino más próximo $=0,709$; puntuación $\mathrm{z}=2,788$, $\mathrm{p}$-valor $=0,005$.
}

Trab. Prehist., 76, N. ${ }^{\circ}$ 2, julio-diciembre 2019, pp. 219-235, ISSN: 0082-5638

https://doi.org/10.3989/tp.2019.12234 

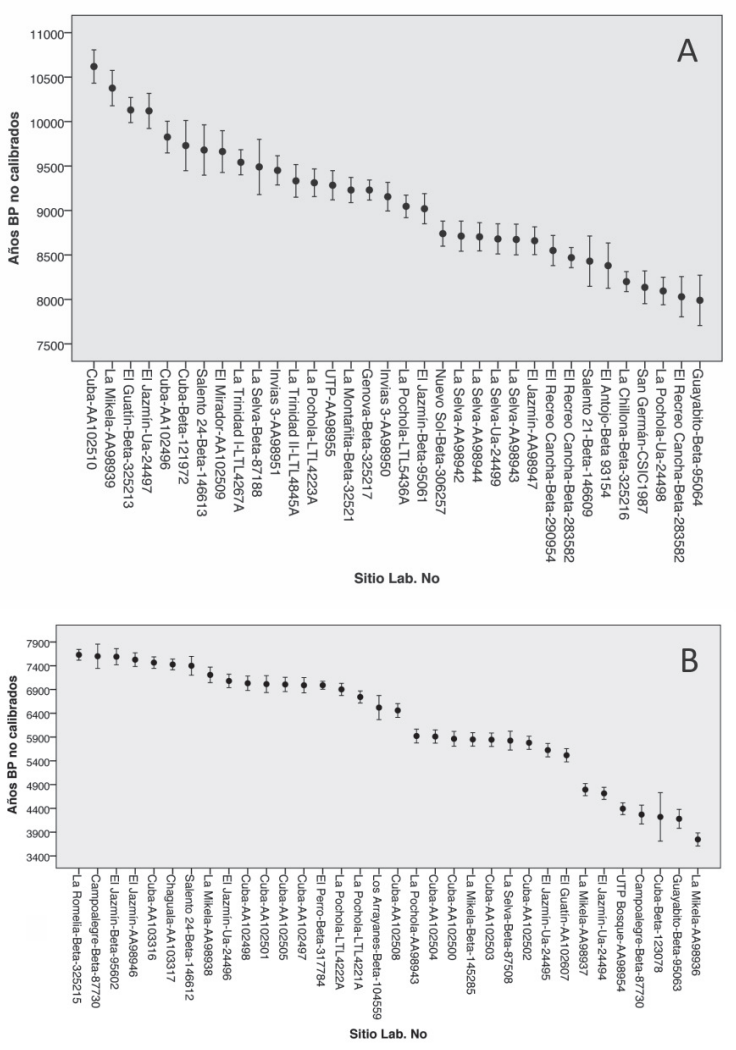

Fig. 3. Gráfico de fechas precerámicas de radiocarbono no calibradas del Cauca medio con dos intervalos de confianza: A. rango cronológico 11.000-7500 BP (finales Pleistoceno/Holoceno temprano). B. rango cronológico 7900-3400 BP (Holoceno medio). El número remite a $\mathrm{AC} 1$.

El primer hecho relevante a este respecto es la ocupación continua desde finales del Pleistoceno hasta aproximadamente el $5400 \mathrm{BP}$. El segundo es que el Cauca medio es una de las regiones del subcontinente con evidencias más tempranas de cultivo de plantas (Aceituno y Loaiza 2014, 2018).

El tema de la movilidad, hasta la fecha, se ha abordado a partir del estudio de la tecnología lítica y la comparación de las características de los sitios arqueológicos (Aceituno y Castillo 2005; Aceituno y Loaiza 2007, 2010) poniendo a prueba el modelo forrajero vs. colector.

Los 25 sitios analizados son contextos con secuencias estratigráficas formadas por diferentes ocupaciones, cuyas dataciones van desde el $10.619 \pm 66 \mathrm{BP}$ (AA102510) al $3746 \pm 49$ BP (AA98936) (Dickau et al. 2015) (Fig. 3; Anexo AC1). Por esta razón no se ha tenido en cuenta la discriminación por períodos a la hora de modelar las rutas, optando por asumir que los sitios, vistos en conjunto, representan un patrón de asentamiento válido para todo el período precerámico. Además de compartir un marco cronológico, la estructura arqueológica de los sitios es similar en cuanto al tamaño, el patrón de descarte, la tecnología lítica y los restos vegetales recuperados. Tales elementos, además de relacionar culturalmente los sitios, indican que los responsables de dicho registro compartieron elementos culturales, ecológicos y subsistenciales. En este sentido, la diferencia altitudinal, que no es muy marcada, a priori no está vinculada con un modelo de complementariedad económica (Aceituno y Loaiza 2007; Loaiza y Aceituno 2015).

Exceptuando el sitio El Antojo (5), un taller lítico de cuarzo, los yacimientos son campamentos residenciales, con diferencias en el tamaño y densidad de artefactos líticos registrados por unidad excavada. La densidad puede considerarse baja respecto a la de otros sitios andinos colombianos (Aceituno y Loaiza 2007: 80). Sus estratigrafías abarcan amplios rangos cronológicos, formados principalmente por desechos de talla de materias primas locales (principalmente andesita y basalto), manos y molinos planos. Entre los escasos instrumentos destacan azadas y artefactos expeditivos. En El Jazmín (7) y La Pochola (9) se identificaron áreas de fogones mezcladas con manos y desechos de talla (Aceituno y Loaiza 2017: 71; Aceituno 2019: 204). Hasta la fecha no se han hallado restos de animales en ningún contexto y los macrorrestos botánicos son escasos. Destacan algunas semillas de palma procedentes de El Jazmín (7) asociadas a niveles del Holoceno medio (Aceituno y Loaiza 2007: 46).

La ausencia de áreas funcionales claramente delimitadas y de evidencias de almacenamiento, la baja densidad de los artefactos líticos, más la ausencia de sitios logísticos, han sugerido un modelo de movilidad residencial (Aceituno y Castillo 2005; Aceituno y Loaiza 2007: 79, 2010). Este se basa en áreas de explotación diaria, que son más comunes en regiones tropicales con una mayor dependencia del consumo de plantas (Kelly 1995: 120; Binford 2001: 276).

Las diferencias de tamaño entre los sitios pueden explicarse como efecto de la variabilidad en la intensidad de uso, el tiempo de ocupación y el número de personas que los habitaron (Binford 2001: 307). Hacia el Holoceno medio, el desarrollo de un modo de vida horticultor, basado en el cultivo de plantas como el maíz (Zea mays), la mandioca (Manihot esculenta) y el fríjol (Phaseolus vulgaris), debió de ajustar los pulsos de la movilidad (Aceituno y Loaiza 2007, 2014, 2018). Este punto no es muy claro, dado que no se advierten cambios significativos en los sitios coincidentes con la aparición de cultígenos en el registro arqueológico. Sea como fuere, todo indica que la movilidad residencial fue el modelo que se mantuvo como 
la principal estrategia de ocupación hasta por lo menos finales del Holoceno medio. Por supuesto, en ningún caso se niega que variara, p. ej., la duración de los campamentos y, por lo tanto, la intensidad de los cambios residenciales anuales.

\section{METODOLOGÍA: ESTUDIO DE LA MOVILIDAD MEDIANTE EL ANÁLISIS DE SUPERFICIES DE COSTE}

El modelado de la movilidad y las rutas de comunicación se ha llevado a cabo mediante análisis $\mathrm{SIG}^{2}$, concretamente a través del "análisis de superficies de coste" o "superficies de fricción" (resumen su aplicación en arqueología Wheatley y Gillings 2002: 151159; Conolly y Lake 2006: 252-256; Grau 2011; Fábrega-Álvarez 2016; y algunos trabajos en Bell et al. 2002; Bermúdez 2006; Fábrega-Álvarez 2006; Fairén et al. 2006; Carballo y Pluckhah 2007; Fábrega-Álvarez y Parcero-Oubiña 2007; Howey 2007; Aguilella 2011; Llobera et al. 2011; Murrieta-Flores et al. 2011; Murrieta-Flores 2012; Magnin et al. 2012; Martínez y Martorell 2012; White y Barber 2012; Verhagen 2013; Güimil-Fariña y Parcero-Oubiña 2015; Bruggencate et al. 2016; Byrd et al. 2016; Villaverde et al. 2016). El análisis de superficies de coste elabora modelos sobre movilidad a partir del estudio de los factores paisajísticos que la condicionan. El presente estudio no pretende reconstruir los caminos antiguos, sino esbozar con carácter exploratorio potenciales rutas de comunicación que puedan relacionarse con el patrón de asentamiento.

La capa básica es la "superficie de coste". Representa la distribución espacial de la variable de coste, la cual cuantifica la resistencia diferencial al movimiento a través del paisaje. Dicha variable suele expresarse en unidades de tiempo o velocidad y se calcula en función de dimensiones relevantes para el desplazamiento, como la topografía, la litología o la cubierta vegetal. La variable usada con más frecuencia es la pendiente topográfica.

El uso de algoritmos basados en la topografía se debe tanto a su significativa influencia en el tránsito como a su relativa estabilidad, al menos a escala general y a largo plazo (Bell et al. 2002). Por supuesto, los cursos de agua o la vegetación influyen al recorrer

\footnotetext{
${ }^{2}$ Se ha empleado el software ArcGIS (v. 10.4). El sistema de coordenadas utilizado es UTM 18 norte, Datum WGS84. Los límites del área analizada son $X$ mínima $=395.000 \mathrm{~m} ; \mathrm{X}$ máxima $=465.000 \mathrm{~m} ; \mathrm{Y}$ mínima $=490.000 \mathrm{~m} ;$ Y máxima $=575.000 \mathrm{~m}$. Las herramientas SIG empleadas, tomadas del paquete ArcGIS-ArcToolbox, se citan en notas al pie. Para automatizar la ejecución de un mismo proceso para $\mathrm{n}$ elementos, se ha recurrido al ModelBuilder, incluido en el propio paquete ArcGIS.
}

el paisaje. Igualmente, factores sociales, como las fronteras, o ideológicos, como los tabúes o la sacralización del paisaje, que no han dejado marcas fáciles de identificar arqueológicamente, también condicionan las rutas seguidas por la gente.

Para la elaboración de la superficie de coste, hemos utilizado exclusivamente la pendiente topográfica, asumiendo esta como condicionante fundamental del desplazamiento. Otro factor importante como los cursos de agua, en nuestro caso son de orden menor, al ser fácilmente vadeables en la mayoría de las condiciones. La capa se ha elaborado como sigue:

1. Obtención del modelo digital de elevaciones (MDE) con el que se creará la capa de pendientes. El MDE usado es el SRTM DEM, creado por la NASA ${ }^{3}$, en concreto la versión procesada y distribuida por el Consortium for Spatial Information (CGIAR) ${ }^{4}$ (Jarvis et al. 2008), con una resolución espacial de 3 segundos sexagesimales, lo que equivale a unos $90 \mathrm{~m}$.

2. Elaboración de la capa de pendientes, expresadas en porcentaje, a partir del $\mathrm{MDE}^{5}$.

3. Elaboración de la capa de costes (Fig. 4A) a partir de la de pendientes. La variable de coste utilizada en este trabajo es el inverso de la velocidad o, en otros términos, la "lentitud", y se basa en el algoritmo formulado por Gorenflo y Gale (1990: 244), que calcula la velocidad de marcha a partir de la pendiente del terreno.

$$
v=6 e^{-3.5|s+0.05|}
$$

Donde $v$ es la velocidad (en $\mathrm{km} / \mathrm{h}$ ), $s$ es la pendiente (en tanto por uno, esto es, la tangente del ángulo) y $e$ es la base del logaritmo natural. La velocidad se calcula mediante la fórmula citada y, a continuación, se convierte a valores de "lentitud" en seg/m.

\subsection{Sitios arqueológicos y transitabilidad}

Este estudio de la movilidad busca, en primer lugar, explorar el posible vínculo de los sitios arqueológicos con las rutas potenciales de circulación. Para ello hemos elaborado un mapa de movilidad potencial o transitabilidad mediante el análisis MADO. Este método de modelización de la movilidad establece caminos óptimos (rutas de más fácil desplazamiento) desde un punto determinado, a través de un área dada y sin un destino concreto (formulación completa en FábregaÁlvarez 2006 y aplicaciones arqueológicas en FábregaÁlvarez y Parcero-Oubiña 2007; Parcero-Oubiña et al.

\footnotetext{
3 Shuttle Radar Topography Mission: http://www2.jpl.nasa.gov/srtm/.

${ }^{4}$ CGIAR-CSI SRTM 90m Digital Elevation Data:

http://srtm.csi.cgiar.org/.

5 ArcGIS-ArcToolbox-Surface-Slope.
} 

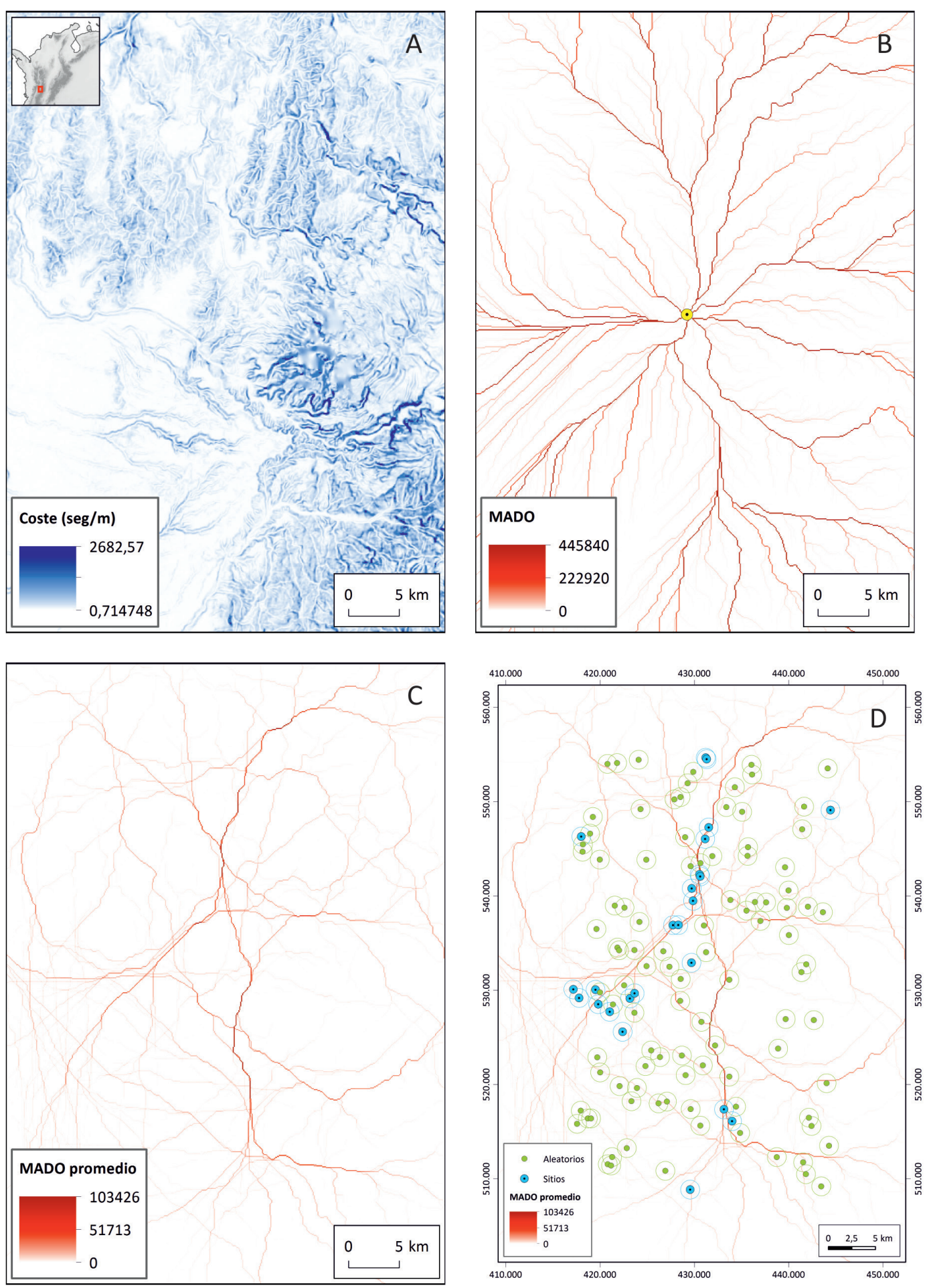

Fig. 4. Zona de estudio (Cauca Medio, Colombia): A. Capa de costes, expresados en segundos por metro. B. Ejemplo de capa Modelo de Acumulación del Desplazamiento Óptimo (MADO) y C. Capa de transitabilidad (MADO promedio); las escalas cromáticas representan, de forma continua, el grado de accesibilidad o movilidad en cada posición, desde el valor mínimo (cero), en color blanco, hasta sus valores más altos, en los tonos más oscuros. D. Sitios arqueológicos y muestra aleatoria de localizaciones, con sus respectivas áreas circulares de $1 \mathrm{~km}$ de radio, sobre el MADO promedio (en color en la versión electrónica). 
2009; Fábrega-Álvarez et al. 2011). Combina el análisis de superficies de coste con el modelado hidrológico para generar una red de rutas de forma dendrítica cuyo ramal principal parte del punto origen y del que van surgiendo ramales al distanciarse de él (Fig. 4B). Una capa MADO vendría a representar las líneas de más fácil tránsito desde un punto de origen concreto. El proceso de elaboración del MADO es el siguiente:

1. Creación de la capa de "coste acumulado" (o "coste-distancia") ${ }^{6}$ desde el punto de origen. Esta capa muestra la distancia, en unidades de coste (p. ej., tiempo), entre el punto de origen y cada punto del área de estudio.

2. Generación de la capa "dirección del flujo"7 a partir de la capa de coste acumulado.

3. Generación de la capa MADO (Fig. 4B) mediante la herramienta de "acumulación del flujo". En la capa resultante cada posición del área de estudio tiene un valor de acumulación que expresa el grado de accesibilidad de dicha posición. Este valor es máximo en el punto de origen y va disminuyendo a medida que nos alejamos de él.

El MADO modela las rutas más fácilmente transitables desde un punto concreto (una aplicación para el ámbito colombiano en Aceituno y Uriarte 2018). Para valorar la movilidad general en el Cauca ${ }^{9}$ hemos superpuesto MADO tomados desde varios puntos, siguiendo a sus propios formuladores (Parcero-Oubiña et al. 2009; Fábrega-Álvarez et al. 2011; FábregaÁlvarez 2016: 177-181). El resultado es un mapa de transitabilidad (Fig. 4C), esto es, de las rutas más fácilmente practicables en el conjunto del área de estudio, elaborado como sigue:

1. Creación de la capa de puntos desde los que vamos a generar los MADO. Dicha capa es una malla ortogonal de puntos, lo suficientemente densa como para ser una muestra representativa de los posibles puntos de origen en el área de estudio ${ }^{10}$.

2. Generación del MADO de cada punto de la malla.

\footnotetext{
${ }^{6}$ ArcGIS-ArcToolbox-Spatial Analysis Tools-Distance-Cost Distance.

7 ArcGIS-ArcToolbox-Spatial Analysis Tools-Hydrology-Flow Direction.

${ }^{8}$ ArcGIS-ArcToolbox-Spatial Analysis Tools-Hydrology-Flow Accumulation.

9 Ver algunas aproximaciones en la península ibérica y México en Bermúdez 2006; Fairén et al. 2006; Murrieta et al. 2011; MurrietaFlores 2012; White y Barber 2012.

${ }_{10}$ Características de la malla ortogonal: El paso de malla es de 2500 $\mathrm{m}$ en longitud y latitud. Los límites (en coordenadas UTM 18 norte, Datum WGS84) son X mínima $=410.000 \mathrm{~m} ; \mathrm{X}$ máxima $=450.000 \mathrm{~m}$; $\mathrm{Y}$ mínima $=505.000 \mathrm{~m}$; Y máxima $=560.000 \mathrm{~m}$. Ello resulta en un total de $17 \times 23$ puntos $(n=391)$.
}

3. A partir de los MADO generados se crea una capa resumen que expresa la movilidad potencial en el área de estudio, la capa de transitabilidad (Fig. 4C). Hemos calculado el promedio de todos los MADO $(\mathrm{n}=391)^{11}$. El resultado es una capa en la que los valores altos "dibujan" una red de rutas preferentes, mientras que los valores bajos se distribuyen por el resto del área de estudio, en los espacios entre dichas rutas.

Después se analiza la relación del MADO promedio con la capa de yacimientos para valorar la mayor o menor vinculación de estos a las rutas potenciales. Para ello se ha cuantificado la transitabilidad del entorno de los yacimientos comparándola estadísticamente ${ }^{12}$ con una muestra de control, formada por ubicaciones aleatorias (Fig. 4D).

Primero se genera una capa con 100 puntos aleatorios dentro del área rectangular mínima que enmarca los 25 yacimientos ${ }^{13}$ y después un área circular de $1 \mathrm{~km}$ de radio en torno a cada localización ${ }^{14}$ (yacimientos y puntos aleatorios). Se calcula la transitabilidad media de cada área circular, a partir de la capa MADO promedio, tabulando los valores resultantes de cara al análisis estadístico ${ }^{15}$. El valor resultante expresa la idoneidad de un área concreta para ser recorrida; cuanto mayor sea, más fácil será el tránsito. En nuestro caso, hemos calculado la transitabilidad media del entorno próximo de cada localización (yacimientos y puntos aleatorios), entendiendo como "entorno próximo" la citada área circular de $1 \mathrm{~km}$ de radio.

Al final los valores de transitabilidad media de los yacimientos se comparan con los de los puntos aleatorios.

\subsection{Caminos óptimos y distancias}

El análisis MADO ha sido complementado con la exploración de la movilidad entre sitios arqueológicos mediante el cálculo del "coste-distancia" y el trazado de "caminos óptimos" entre yacimientos (Fig. 5).

El "coste-distancia" (o "coste acumulado") es la distancia, en unidades de coste (en nuestro caso, unidades de tiempo), que separa una ubicación de otra.

\footnotetext{
11 ArcGIS-ArcToolbox-Spatial Analysis Tools-Local-Cell Statistics

12 Se ha empleado el software IBM SPSS Statistics (v. 24).

13 ArcGIS-ArcToolbox-Data Management Tools-Feature Class-Create Random Points. La aleatoriedad de la muestra se ha contrastado mediante el análisis del vecino más próximo (ArcGIS-ArcToolbox-Spatial Statistics Tools-Analyzing Patterns-Average Nearest Neighbor), con resultados positivos: índice del vecino más próximo $=1,064$; puntuación $\mathrm{z}=1,225$, p-valor $=0,221$.

${ }^{14}$ ArcGIS-ArcToolbox-Spatial Analysis Tools-Proximity-Buffer.

15 ArcGIS-ArcToolbox-Spatial Analysis Tools-Zonal-Zonal Statistics as Table.
} 

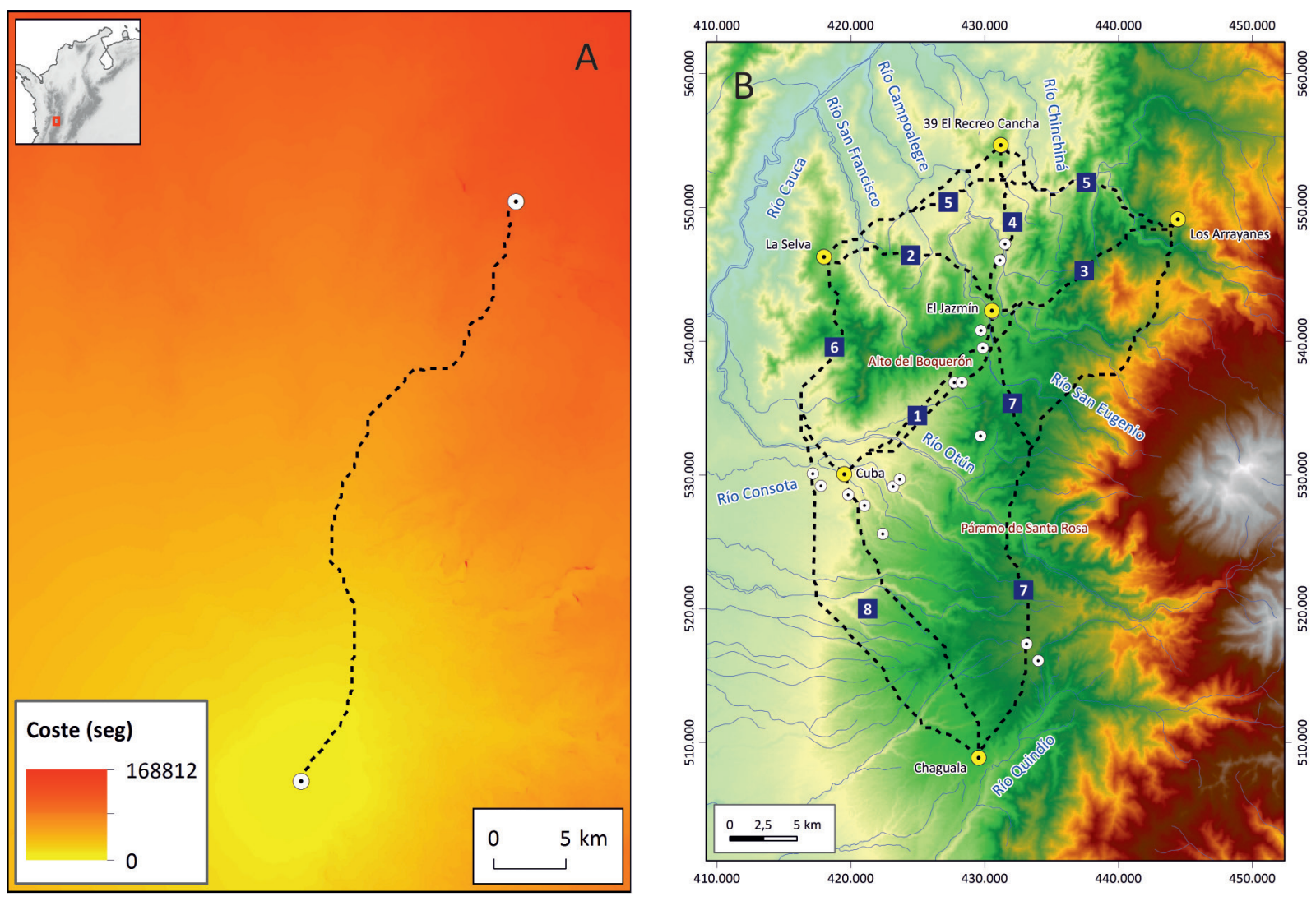

Fig. 5. Zona de estudio (Cauca medio, Colombia): A. Ejemplo de capa de coste-distancia o coste acumulado (desde el sitio núm. 25 Chaguala) y de camino óptimo entre dos localizaciones (desde Chaguala al sitio núm. 4 Los Arrayanes). B. Red de caminos óptimos, tomados desde seis sitios representativos. Cartografía base: CGIAR-CSI SRTM 90m Digital Elevation Data (Jarvis et al. 2008); Hidrografía de Colombia, Instituto Geográfico Agustín Codazzi (en color en la versión electrónica).

Es decir, es el tiempo mínimo necesario para llegar de una localización a otra y, por ende, el que se emplea en recorrer el camino óptimo. Se obtiene a través de la capa de coste-distancia ${ }^{16}$, que muestra la distancia, en unidades de coste (p. ej., tiempo), que hay de un punto dado de origen al resto de localizaciones del área de estudio.

El "camino óptimo", o "ruta de menor coste"17, es el que une una localización con otra con el menor coste-distancia posible. Su representación cartográfica es una línea irregular, la cual simula un trayecto. Para representar de modo más claro y sencillo las rutas potenciales, y basándonos en el patrón concentrado de distribución de los 25 yacimientos estudiados, hemos seleccionado 6 representativos de otras tantas áreas y franjas altitudinales (Fig. 5B). Dichas áreas, establecitance.

${ }^{16}$ ArcGIS-ArcToolbox-Spatial Analysis Tools-Distance-Cost Dis-

${ }^{17}$ ArcGIS-ArcToolbox-Spatial Analysis Tools-Distance-Cost Path. das de visu, están vinculadas a las subcuencas hidrográficas del río Cauca: San Francisco, Chinchiná, Otún, Consota, La Vieja y Quindío.

\section{RESULTADOS}

El trazado de rutas resultado del análisis MADO indica una clara relación entre las zonas de mayor transitabilidad y la ubicación de los sitios arqueológicos (Fig. 4D). La comparación mediante un diagrama de caja (Fig. 6A) muestra, en líneas generales, valores más altos para los sitios arqueológicos. La diferencia estadísticamente significativa entre ambos grupos, según la prueba de Mann-Whitney (Tab. 1), corrobora esta observación. Además, solo La Selva (1), El Recreo Cancha (2), El Perro (3), Campoalegre (13) y El Guatín (19) tienen valores inferiores a la mediana de los puntos aleatorios, esto es, pueden considerarse poco accesibles, mientras que los otros 20 la superan. Es 

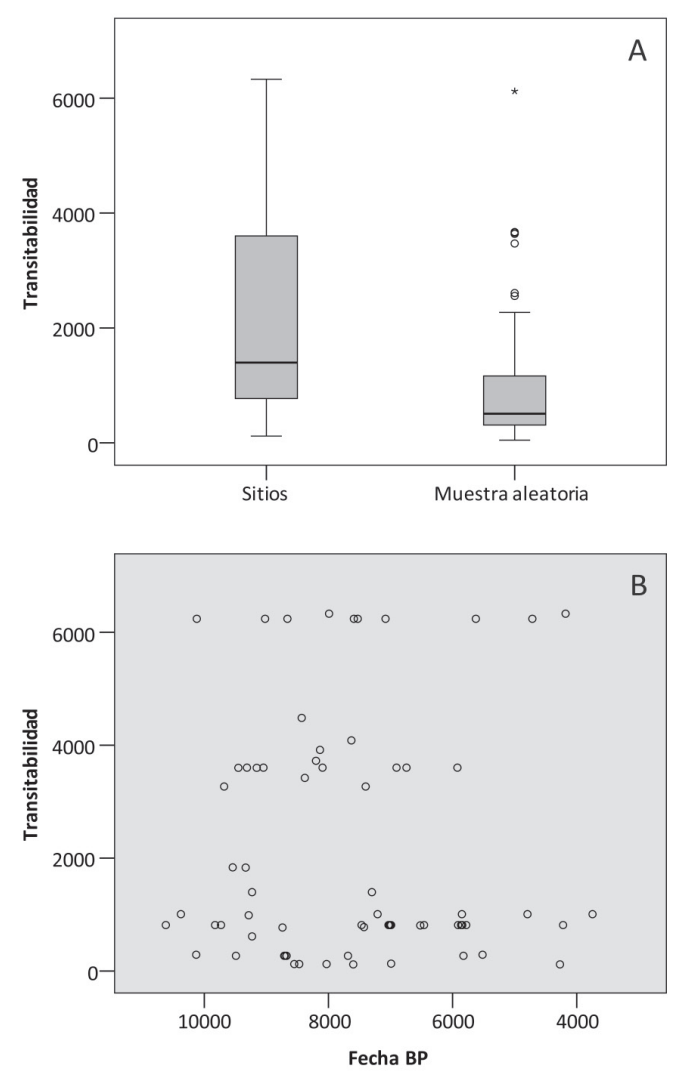

Fig. 6. Zona de estudio (Cauca medio, Colombia): A. Diagrama de caja donde se comparan los valores de transitabilidad de los sitios arqueológicos con los de los puntos aleatorios. B. Diagrama de dispersión donde se relacionan las fechas BP de los sitios arqueológicos con sus correspondientes valores de transitabilidad.

decir, el resultado del análisis estadístico apoya la hipótesis de una cierta vinculación entre la ubicación de los yacimientos y las áreas mejor comunicadas.

Con el fin de evaluar la posible variación temporal de tal vinculación, hemos explorado la relación de los valores de transitabilidad de los sitios arqueológicos con sus dataciones radiocarbónicas. El gráfico de dispersión de los valores medios de las fechas BP versus los valores medios de transitabilidad de las áreas de 1 $\mathrm{km}$ de los 25 yacimientos (Fig. 6B), no parece mostrar variación alguna en función de la cronología. El análisis de regresión (Tab. 2) refuerza de manera concluyente esta idea, al mostrar una relación prácticamente inexistente.

La figura 5B muestra el mapa con las rutas de menor coste generadas y la tabla 3 los costes medidos en tiempo y distancia entre pares de sitios. Destaca la ruta 1 , que conecta los sitios localizados en el valle del río San Eugenio (cuenca Campoalegre) con los contextos ubicados en el abanico Pereira-Armenia,

\begin{tabular}{|c|c|c|c|c|}
\hline & GRUPO & $\mathbf{N}$ & $\begin{array}{c}\text { Rango } \\
\text { promedio }\end{array}$ & $\begin{array}{c}\text { Suma } \\
\text { de } \\
\text { rangos }\end{array}$ \\
\hline \multirow{3}{*}{ Transitabilidad } & Sitios & 25 & 83,72 & 2093,00 \\
\hline & $\begin{array}{l}\text { Muestra } \\
\text { aleatoria }\end{array}$ & 100 & 57,82 & 5782,00 \\
\hline & Total & 125 & & \\
\hline \multicolumn{5}{|c|}{ Transitabilidad } \\
\hline \multicolumn{3}{|c|}{ U de Mann-Whitney } & & 732,000 \\
\hline \multicolumn{3}{|l|}{ W de Wilcoxon } & & 5782,000 \\
\hline \multicolumn{3}{|l|}{$\mathrm{Z}$} & & $-3,197$ \\
\hline \multicolumn{3}{|c|}{ Sig. asintótica (bilateral) } & & 001 \\
\hline
\end{tabular}

Tab. 1. Prueba U de Mann-Whitney: Comparación de los valores de transitabilidad de los sitios arqueológicos con los de los puntos aleatorios.

entre el río Otún y Consota. Esta ruta aprovecha un corredor natural que cruza una línea de cumbres, asociadas a la curva de nivel de los $2.000 \mathrm{~m} \mathrm{~s}$. n. m., que separa ambos pisos altitudinales. Esta ruta cruza dicha línea de cumbres por el Alto del Boquerón a $1.774 \mathrm{~m}$ s. n. m., entre el Alto de la Cruz y el Alto Vázquez, y desciende hasta los contextos del río Consota en la actual ciudad de Pereira, a una altitud entre los 1.250 y los $1.500 \mathrm{~m} \mathrm{~s}$. n. m. Este recorrido aprovecha este paso natural donde la sierra es más baja, conectando tres cuencas hidrográficas (Campoalegre, Otún y Consota) con la "suela plana" del río Cauca (900 m s. n. $\mathrm{m}$.), donde por el momento no se han reportado sitios arqueológicos precerámicos. Esta gran ruta se bifurca antes del Alto del Boquerón, en el gran flujo de depó-

\begin{tabular}{|r|r|r|r|}
\hline \multicolumn{1}{|r|}{$\mathbf{R}$} & \multicolumn{1}{|c|}{$\begin{array}{c}\text { R } \\
\text { cuadrado }\end{array}$} & $\begin{array}{c}\text { R } \\
\text { cuadrado } \\
\text { ajustado }\end{array}$ & $\begin{array}{c}\text { Error } \\
\text { estándar de } \\
\text { la estimación }\end{array}$ \\
\hline, 009 &, 000 &,- 015 & 2132,445186 \\
\hline
\end{tabular}

Predictores: (Constante), Fecha BP

Variable dependiente: Transitabilidad

\begin{tabular}{|c|c|r|r|r|r|}
\cline { 2 - 6 } \multicolumn{1}{c|}{} & \multicolumn{2}{|c|}{$\begin{array}{c}\text { Coeficientes no } \\
\text { estandarizados }\end{array}$} & $\begin{array}{c}\text { Coeficientes } \\
\text { estandariza- } \\
\text { dos }\end{array}$ & t & Sig. \\
\cline { 2 - 6 } & \multicolumn{1}{c|}{ B } & $\begin{array}{c}\text { Error } \\
\text { estándar }\end{array}$ & Beta & & \\
\hline (Cons.) & 2067,725 & 1219,153 & & 1,696 &, 095 \\
\hline $\begin{array}{c}\text { Fecha } \\
\text { BP }\end{array}$ &,- 011 &, 156 &,- 009 &,- 070 &, 945 \\
\hline
\end{tabular}

Tab. 2. Análisis de regresión: Relación de las fechas BP de los sitios arqueológicos con sus correspondientes valores de transitabilidad. 
sitos volcánicos donde actualmente se sitúa la ciudad de Santa Rosa de Cabal. Desde allí desciende por un valle, a unos $1.700 \mathrm{~m} \mathrm{~s}$. n. m., delimitado por la cima de La Paloma y el Alto del Toro, hasta llegar al río Otún. A partir de ahí, se atraviesa el piedemonte del Páramo de Santa Rosa por la franja altitudinal entre los 1.700 y los $1.800 \mathrm{~m}$ s. n. m. hasta llegar al río Quindío, que comunica con el sitio Chaguala (25), el más meridional del área de estudio. Otras rutas importantes son (Fig. 5B):

a) Ruta 2: Enlaza los sitios del río San Eugenio con el de La Selva (1), que transcurre por el valle del río San Francisco, afluente del río Cauca, localizado al norte del río Otún.

b) Ruta 3: Va desde el valle del río San Eugenio (a unos $1.650 \mathrm{~m} \mathrm{~s}$. n. m. de altitud) hasta las tierras altas del sitio Los Arrayanes (4) (2.275 m s. n. m.): asciende por el valle del río Campoalegre y cruza el río Claro y pequeños valles de afluentes del río Chinchiná hasta llegar al yacimiento.

c) Ruta 4: Conecta el valle del río San Eugenio con los sitios El Recreo Cancha (2) y El Perro (3) (cuenca Chinchiná), a través de los valles de los ríos Campoalegre y Chinchiná.

d) Ruta 5: Entre Los Arrayanes (4) y La Selva (1), desciende por el valle del río Chinchiná, cruza el del río Campoalegre, recorre una pequeña meseta hasta llegar al valle del San Francisco (1.150 m s. n. m.). Desde allí asciende hasta la cuchilla donde está La Selva (1) (1.577 m s. n. m.).

e) Ruta 6: Entre La Selva (1) y los sitios del río Consota, desciende por valles estrechos, cruzando por vados naturales la cuchilla Chaquiro y la cima del Rayo, hasta llegar a la altura del río Otún a unos 1.280 m s. n. m., desde donde se alcanza la planicie ondulada donde se localizan los sitios más próximos al río Consota.

f) Ruta 7: Va desde el valle del río San Eugenio hasta los sitios del río Quindío. Transcurre por un pequeño valle a unos $1.700 \mathrm{~m} \mathrm{~s}$. n. m., delimitado por la cima de La Paloma y el Alto del Toro. Al llegar al río Otún $(1.770 \mathrm{~m} \mathrm{~s} . \mathrm{n}$. m.) se atraviesa el piedemonte del Páramo de Santa Rosa por la franja altitudinal entre los 1.700 y los $1.800 \mathrm{~m} \mathrm{~s}$. n. m.

g) Ruta 8: Desde el sitio Chaguala (25) hasta los del río Consota, esta ruta cruza en dirección surestenoroeste la planicie del abanico Pereira-Armenia — con múltiples quebradas afluentes del río La Vieja-, como un corredor altitudinal que baja desde los $1.768 \mathrm{~m} \mathrm{~s}$. n. m. del sitio Chaguala (25) a las altitudes, ya señaladas, del valle del río Consota.

La figura 5B muestra la coincidencia de los grandes clusters en una gran ruta que va desde El Recreo Cancha (2) y El Perro (3) hasta los sitios del abanico fluvio-volcánico Pereira-Armenia, antesala de las tie- rras bajas de la "suela plana" del valle del río Cauca. Son su eje central los valles de los ríos San Eugenio y Campoalegre y el paso natural desde el Alto del Boquerón al abanico Pereira-Armenia. Por esta ruta, a la cual están asociados la mayoría de los yacimientos, transcurre la carretera Panamericana que conecta la Cordillera Central con el valle del río Cauca, siendo una de las rutas actuales más importantes que conecta en dirección norte-sur los Andes colombianos.

\section{DISCUSIÓN}

Ya hemos señalado en la introducción el gran desarrollo del estudio de las rutas de movilidad y la creciente aplicación de los SIG a través del análisis de costes. Muchos de estos trabajos -entre los que se encuentra el aquí presente- se han centrado en regiones montañosas, cuyo paisaje, especialmente la topografía, condiciona la movilidad de manera crucial.

Dos han sido las herramientas metodológicas empleadas (Fig. 7). La primera es el trazado de caminos óptimos, una herramienta SIG convencional muy frecuentemente usada en los estudios arqueológicos de movilidad. La segunda es el análisis MADO, diseñado en el seno de la propia investigación arqueológica y con menor difusión hasta la fecha, aunque con un gran potencial. Mientras los caminos óptimos vinculan pares de localizaciones, el análisis MADO relaciona una localización con el conjunto del área. Asimismo, la combinación de varios MADO, tomados desde diferentes puntos, nos ha permitido aproximarnos a la estructura general de movilidad de la región y, después, analizar la ubicación de los sitios arqueológicos en relación con esa estructura.

La red de rutas establecidas mediante los caminos óptimos y el análisis MADO suma elementos al paisaje arqueológico del Cauca medio que aportan claves para entender el territorio de esta región andina desde la movilidad y transitabilidad. La prolongada ocupación de los sitios analizados en el texto da cuenta de las posibilidades del territorio para una próspera cultura de cazadores-recolectores y cultivadores incipientes de selvas de montaña. Sus redes de caminos formarían parte de un territorio con diferentes escalas espaciales de movilidad y de interacciones inter-grupales que, por el momento, desconocemos. Las áreas donde se congregan los sitios arqueológicos, de alguna forma representan un patrón de asentamiento asociado con locaciones estratégicas para aprovechar los recursos medioambientales y mantener interacciones intergrupales.

Tales caminos permitirían el tránsito de personas, ideas y sobre todo la interacción entre los grupos o unidades sociales autónomas que configurarían el pai- 


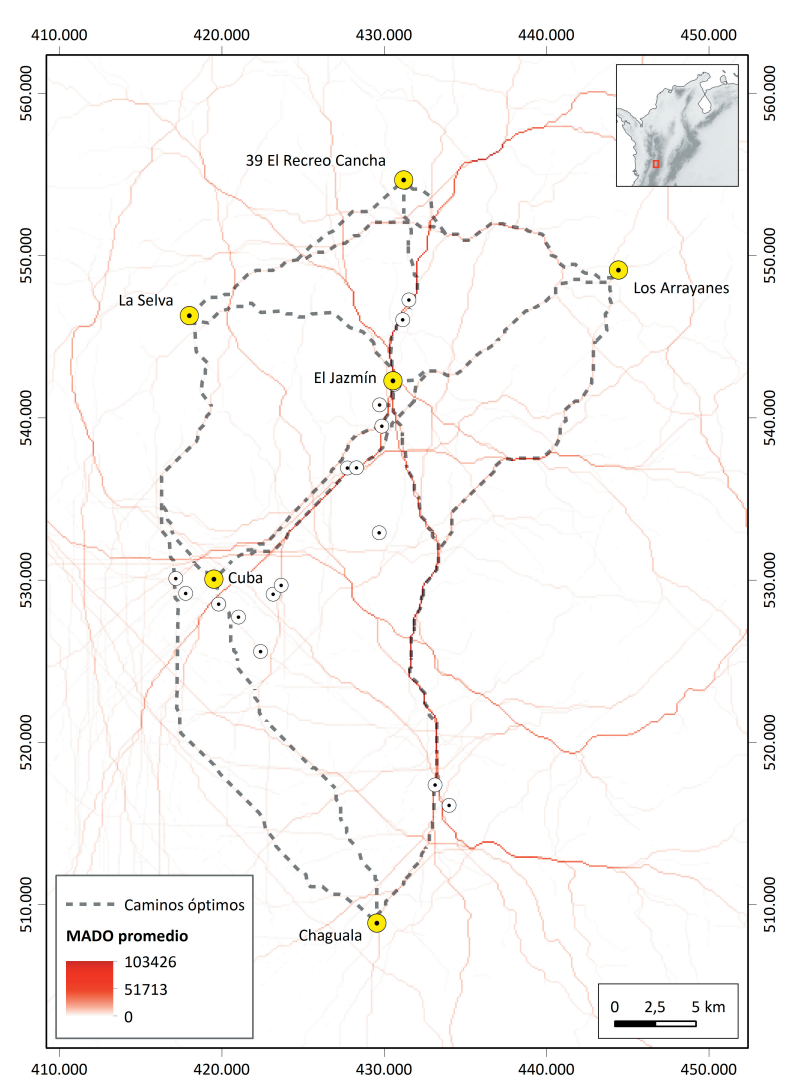

Fig. 7. Zona de estudio (Cauca medio, Colombia): Visualización conjunta de la capa de transitabilidad (Modelo de Acumulación del Desplazamiento Óptimo promedio) y la red de caminos óptimos (en color en la versión electrónica).

saje. Las rutas ayudan a optimizar los contactos entre la gente, la búsqueda de recursos y el traslado de unas zonas a otras, en una estrategia itinerante de ocupación del territorio. Las características de los sitios arqueológicos se corresponden con un modelo de movilidad residencial con reocupación de los sitios habitacionales, como indican las secuencias estratigráficas de los sitios intervenidos. Esto indica que el modelo se mantuvo durante milenios en esta zona montañosa del Cauca medio.

Los caminos trazados (MADO, análisis de mínimo coste) conectarían los movimientos entre campamentos residenciales y entre estos y la explotación del cuarzo lechoso del sitio El Antojo (5). Como no sabemos cuántos campamentos formarían parte de un ciclo anual, es difícil calcular las distancias promedio a partir del registro arqueológico. Algunos datos etnográficos pueden servir de referente. P. ej., entre los NukakMakú (Amazonía colombiana), la distancia promedio entre campamentos residenciales es de $7,7 \mathrm{~km}$ y se han registrado entre 55 y 68 movimientos al año (Cárdenas y Politis 2000: 34; Binford 2001: 270). En otros grupos que habitan en diferentes regiones tropicales del planeta, se ha calculado una distancia promedio alrededor de unos 8,8 km (Siriono, Agta, Batak, Aka, Vedda, Mbuti y Semang) (Kelly 1995: 114).

Desde un punto de vista arqueológico, definir o estimar la intensidad de los movimientos es una tarea ardua. Según el principio de optimización que rige la teoría del modelo de óptimos forrajeros (Cooding y Bird 2016; Stiner y Khuhn 2016), los grupos que practican movilidad residencial deben cambiar de campamento cuando los costes de obtención de alimentos, por unidad de tiempo, igualan o incluso superan los beneficios. Entonces, es más eficiente cambiar de campamento residencial que alargar el radio del área de captación de recursos, que implica más tiempo en la adquisición y en el transporte de los recursos a los campamentos residenciales (Morgan 2009; Venkataraman et al. 2017). Para estimar la duración de los campamentos se deben tener datos sobre ubicación de recursos y costes de obtención. Sin dichos cálculos, principalmente por ausencia de datos, sí se puede plantear que la intensificación de la producción, mediante la horticultura, alterara de algún modo el pulso de la movilidad, reduciéndola en proporción directa a la producción de alimentos, como sugieren los datos etnográficos (Binford 2001: 189). Un dato importante a considerar es que la aparición de la horticultura en la zona coincide con la máxima expresión del óptimo climático entre el 7000 y el $6000 \mathrm{BP}$, un período más cálido y húmedo con temperaturas entre 1 y $2{ }^{\circ} \mathrm{C}$ superiores a las actuales (Melief 1985). La duración de los campamentos en grupos contemporáneos varía entre la alta movilidad de los Nukak-Makú, o una movilidad anual más restringida. El promedio de los movimientos residenciales anuales en los grupos antes citados es de 14,85 (Kelly 1995: 114).

En términos altitudinales la zona de estudio está entre los 1.200 y $2.300 \mathrm{~m} \mathrm{~s}$. n. m. La ausencia de estaciones térmicas en el Neotrópico y la homogeneidad en la distribución de los recursos bióticos (entre los 1.000 y los $2.800 \mathrm{~m} \mathrm{~s}$. n. m., pisos templado y frío) implica una modalidad residencial muy diferente a la de las zonas templadas, donde operan modelos de complementariedad altitudinal según la estación térmica (Morgan 2009; García-Moreno et al. 2012).

En términos ambientales el manejo altitudinal estaría más marcado por la localización de áreas de recursos y el grado de agotamiento de recursos o de buenos suelos para el cultivo de plantas, que por una distribución altitudinal de recursos estratégicos.

Igualmente, el uso de materias primas locales para la manufactura de artefactos excluye la hipótesis del abastecimiento de rocas como determinante de la mo- 
vilidad. Únicamente a partir de la cota de los $2.800 \mathrm{~m}$ s. n. m., que inicia la transición hacia el páramo, cambian mucho la fauna y, sobre todo, la flora, desapareciendo los bosques a favor de la vegetación herbácea y arbustiva. Hasta el momento no hay evidencias arqueológicas de la explotación de dicho piso altitudinal en el Macizo Volcánico.

El registro arqueológico no muestra claras evidencias de cambios en el modelo, aunque no descartamos que los hubiera. No varían significativamente ni el tamaño de las ocupaciones, ni el patrón de descarte de la tecnología lítica. Tampoco aparecen formas de almacenamiento como la cerámica o silos que indiquen con más o menos claridad una ocupación más prolongada de los asentamientos. La única evidencia son las huellas de poste registradas en el sitio La Pochola (9) y asociadas a una fecha de $6743 \pm 45$ BP. Indicarían construcciones más sólidas, vinculadas con un cultivo de plantas, que permitiría una reducción de la movilidad (Aceituno 2019: 205). Pero siguen faltando en el paisaje campamentos logísticos asociados con una mayor duración de los campamentos residenciales para aprovechar recursos, más allá del radio de forrajeo diario. Esto sugiere que los grupos que ocuparon la zona, a pesar de la producción de alimentos, mantuvieron la movilidad como estrategia de ocupación del territorio, practicando una horticultura itinerante (Aceituno y Loaiza 2007: 95). La estrategia está registrada en grupos de selva tropical como los Nukak-Makú (Politis 1996), los Hoti (Venezuela) (Politis 2005) o los Huaorani (Amazonía ecuatoriana) (Rival 1999: 102). Esto prueba una vez más que producción de alimentos no se traduce automáticamente en sedentarización. La estabilidad en el registro arqueológico y la permanencia en la región que muestran las fechas de radiocarbono (Fig. 3), indican la adaptación a largo plazo de los grupos a los eventos volcánicos registrados en la zona durante el Holoceno (Dickau et al. 2015). La evolución climática durante el Holoceno tampoco afectó a la configuración de la zona de estudio en términos de cobertura vegetal, como hemos podido observar a través de las columnas de polen de los sitios El Jazmín (7), Campoalegre (13), La Pochola (9) y La Selva (1) (Aceituno y Loaiza 2007: 84; Mercado 201018; Aceituno 2019: 207).

Las áreas de cultivo, con suelos preparados durante generaciones, se convertirían en un recurso espacial muy importante, que probablemente demandó algún tipo de acuerdos o normas sobre derechos de uso en-

\footnotetext{
18 Mercado, J. 2010: Análisis polínico en el yacimiento la Pochola un contexto precerámico en el río San Eugenio (Cauca medio, Risaralda-Colombia-). Tesis de maestría. Instituto de Biología, Facultad de Ciencias Exactas y Naturales, Universidad de Antioquia, p. 122.
}

tre las unidades sociales de la zona. La dispersión de estas áreas en el territorio puede ser vista como una especie de almacenamiento espacial que mejora la predicción de los recursos y su accesibilidad anual, reduciendo el riesgo y la incertidumbre. Este tipo de movilidad tiene ventajas adicionales como posibilitar la recuperación de los suelos y reducir la presión sobre la caza, un recurso crítico que no es muy abundante en términos de biomasa secundaria en los bosques tropicales (Aceituno y Loaiza 2007: 96). En esta estrategia de manejo itinerante del territorio, los caminos jugarían un papel clave en los desplazamientos a los lugares de explotación de los recursos y, probablemente, también al elegir el emplazamiento de los sitios. Esto se observa, p. ej., en los contextos localizados en los valles San Eugenio y Campoalegre, una ruta que, a mayor escala, forma parte de un corredor natural de comunicación entre los valles subandinos, a unos 1.650 m s. n. m. de promedio (piso templado del Macizo Volcánico), con el valle del río Cauca, a unos $900 \mathrm{~m}$ s. n. m. de promedio (piso térmico cálido).

Surgen preguntas sobre la tipología de los potenciales caminos. Sea como fuere, su arquitectura guardaría relación con la intensidad de los intercambios, los medios de transporte utilizados y el volumen de carga involucrada. En el Cauca, los caminos se ajustarían a la escala del desplazamiento, dado que estamos ante sociedades pedestres, que nunca usaron animales para desplazarse de un sitio a otro.

Algunas referencias actuales pueden ser útiles para comprender cómo pudieron señalizar estos caminos. Entre los Nukak una de ellas es la alteración de la vegetación y la concentración de algunos tipos de plantas alrededor de los espacios usados como campamentos o huertos (Cárdenas y Politis 2000: 85-87). La dispersión de semillas alrededor de los caminos pudo ser una estrategia para delimitar zonas de tránsito en el territorio.

La necesidad de compartir también demanda infraestructuras de comunicación. En grupos cazadoresrecolectores, el acto de compartir y la reciprocidad son dos estrategias clave encaminadas a mantener el orden social, mediante la resolución de conflictos y los acuerdos sobre el derecho de uso del territorio. La colaboración entre bandas se materializa en la existencia de redes de intercambio, que definen mejor los límites territoriales de grupos móviles, donde el intercambio de información con grupos vecinos es clave en su supervivencia (Sauvet 2017). La modelización de caminos que hemos presentado es importante para comprender el manejo del espacio de los grupos tempranos que habitaron esta región andina, en la medida en que las rutas formarían parte de la imagen del territorio que cada individuo tendría en su mente y su memorización serviría para mantener vivas las redes de inter- 


\begin{tabular}{|c|c|c|c|c|c|}
\hline & Chaguala & Cuba & El Jazmín & Los Arrayanes & $\begin{array}{l}39 \text { EI Recreo } \\
\text { Cancha }\end{array}$ \\
\hline La Selva & $\begin{array}{r}12 \mathrm{~h} 58 \mathrm{~min} \\
45,70 \mathrm{~km}\end{array}$ & $\begin{array}{r}5 \mathrm{~h} 58 \mathrm{~min} \\
19,61 \mathrm{~km}\end{array}$ & $\begin{array}{r}5 \mathrm{~h} 43 \mathrm{~min} \\
15,86 \mathrm{~km}\end{array}$ & $\begin{array}{r}10 \mathrm{~h} 25 \mathrm{~min} \\
31,47 \mathrm{~km}\end{array}$ & $\begin{array}{r}5 \mathrm{~h} 17 \mathrm{~min} \\
16,44 \mathrm{~km}\end{array}$ \\
\hline $\begin{array}{l}39 \text { EI Recreo } \\
\text { Cancha }\end{array}$ & $\begin{array}{r}14 \mathrm{~h} 17 \mathrm{~min} \\
50,65 \mathrm{~km} \\
\end{array}$ & $\begin{array}{r}8 \mathrm{~h} 30 \mathrm{~min} \\
31,07 \mathrm{~km}\end{array}$ & $\begin{array}{r}3 \mathrm{~h} 47 \mathrm{~min} \\
13,46 \mathrm{~km}\end{array}$ & $\begin{array}{r}6 \mathrm{~h} 12 \mathrm{~min} \\
17,88 \mathrm{~km}\end{array}$ & \\
\hline Los Arrayanes & $\begin{array}{r}16 \mathrm{~h} 04 \mathrm{~min} \\
49,27 \mathrm{~km}\end{array}$ & $\begin{array}{r}11 \mathrm{~h} 42 \mathrm{~min} \\
34,32 \mathrm{~km}\end{array}$ & $\begin{array}{r}7 \mathrm{~h} 16 \mathrm{~min} \\
17,19 \mathrm{~km}\end{array}$ & & \\
\hline El Jazmín & $\begin{array}{r}10 \mathrm{~h} 15 \mathrm{~min} \\
37,14 \mathrm{~km} \\
\end{array}$ & $\begin{array}{r}4 \mathrm{~h} 44 \mathrm{~min} \\
17,61 \mathrm{~km} \\
\end{array}$ & & & \\
\hline Cuba & $\begin{array}{l}7 \mathrm{~h} 18 \mathrm{~min} \\
25,06 \mathrm{~km}\end{array}$ & & & & \\
\hline
\end{tabular}

Tab. 3. Matriz de costes-distancia (en tiempo) y distancias (en $\mathrm{km}$ ) de los caminos óptimos entre seis sitios representativos.

cambio en una territorialidad cuyos límites eran desconocidos.

\section{CONCLUSIONES}

Los datos arqueológicos correspondientes a la margen derecha de la cuenca media del río Cauca (Macizo Volcánico) sugieren la ocupación del territorio por las sociedades a pequeña escala que poblaron esta zona de los Andes colombianos en la transición Pleistoceno/ Holoceno. La secuencia cronológica y las características de los sitios arqueológicos indican que esta región estuvo habitada hasta finales del Holoceno medio por sociedades móviles que vivieron de la caza, la recolección y la horticultura.

El modelado de dichas rutas presentado en este trabajo no trata de reconstruir los caminos del pasado, sino de aportar elementos para entender en clave espacial y territorial el registro arqueológico de esta región andina. Los caminos trazados mediante herramientas SIG representarían movimientos residenciales como estrategia de ocupación del territorio, cuya intensidad y distancia entre campamentos es difícil de calcular. Los datos indican que el modelo de movilidad se mantendría durante milenios, lo que no significa ausencia de cambios, p. ej., en la duración de los campamentos, dado que el desarrollo de la producción de alimentos de alguna manera tuvo que modificar los pulsos de movilidad en la zona.

La movilidad como estrategia de ocupación requiere la fijación o el trazado de rutas en el espacio, como parte de la arquitectura antrópica del territorio. En sociedades nómadas los caminos se convierten en instrumentos anclados en el paisaje que optimizan los cambios residenciales, el aprovechamiento de los recursos y posibilitan manejar los límites territoriales y, especialmente, mantener vivas las redes sociales que viabilizan la supervivencia de una sociedad. Nada nos indica que no fuera así durante una gran parte de la historia del Holoceno, conservada en los sitios arqueológicos del Cauca medio.

\section{AGRADECIMIENTOS}

Los datos utilizados sobre la ubicación de los sitios y las fechas de radiocarbono han sido publicados por investigadores a quienes queremos reconocer sus valiosos aportes a la arqueología del Cauca medio (en orden alfabético): Martha Cano, Ruth Dickau, Leonor Herrera, Nicolás Loaiza, Carlos López, Anthony Ranere, Carlos Restrepo y Michael Tistl (q. e. p. d.). A la empresa INTEGRAL por facilitarnos los informes del proyecto de arqueología preventiva Autopistas del Café. A Alexander Clavijo por facilitarnos el informe correspondiente al Gasoducto de Occidente (INCIVA). Al Instituto Colombiano de Antropología e Historia por facilitarnos el informe correspondiente con la excavación del sitio Los Arrayanes (ECOPETROL). El informe correspondiente a la cita Rojas y Tabares 2000 fue facilitado por Dionalver Tabares. El informe correspondiente a la cita Restrepo 2013 fue consultado en la biblioteca del ICANH. Agradecemos a los evaluadores sus pertinentes comentarios para mejorar la versión final del artículo.

\section{ANEXO: ARCHIVO COMPLEMENTARIO}

En la versión en línea (menú Herramientas del artículo) puede consultarse un archivo complementario en formato Excel, que incluye las referencias de las fuentes inéditas de las que proceden algunas de las dataciones radiocarbónicas que se mencionan en dicho archivo:

$\mathrm{AC} 1$. Sitios arqueológicos y dataciones radiocarbónicas de la zona de estudio (Cauca medio, Colom- 
bia). Datos de altitud: CGIAR-CSI SRTM 90m Digital Elevation Data (Jarvis et al. 2008). Todas las fechas han sido calibradas con el programa Calib Rev 7.0.0 y representan 2 sigmas de probabilidad. Curva de calibración: intCal3.14c.

\section{BIBLIOGRAFÍA}

Aceituno, F. J. 2019: Entre el río y la montaña. Nuevos datos para el poblamiento temprano del Cauca medio colombiano. Facultad de Ciencias Sociales y Humanas, Universidad de Antioquia. Medellín. Colombia.

Aceituno, F. J. y Castillo N. 2005: "Mobility strategies in Colombia's middle mountain range between the early and middle Holocene". Before Farming 2005/2: 1-17. https://doi.org/10.3828/bfarm.2005.2.2

Aceituno, F. J. y Lalinde, V. 2011: "Residuos de almidones y el uso de plantas durante el Holoceno medio en el Cauca medio". Caldasia 33 (1): $1-20$.

Aceituno, F. J. y Loaiza, N. 2007: Domesticación del Bosque en el Cauca Medio Colombiano entre el Pleistoceno Final y el Holoceno Medio. British Archaeological Reports, International Series 1654, Archaeopress. Oxford.

Aceituno, F. J. y Loaiza, N. 2010: "Estructura interna y movilidad en el valle del río San Eugenio en la Cordillera Central de Colombia”. Revista de Arqueología del Area Intermedia 8: 83-120.

Aceituno, F. J. y Loaiza, N. 2014: "Early and Middle Holocene evidence for use of plants and cultivation in the Middle Cauca River Basin, Cordillera Central (Colombia)". Quaternary Science Reviews 86: 4962. https://doi.org/10.1016/j.quascirev.2013.12.013

Aceituno, F. J. y Loaiza, N. 2018: "The origins and early development of plant food production and farming in Colombian tropical forests". Journal of Anthropological Archaeology 49: 161-172. https://doi.org/10.1016/j.jaa.2017.12.007

Aceituno, F. J.; Loaiza, N.; Delgado, E. y Barrientos, G. 2013: "The Initial Human Settlement of Northwest South America during the Pleistocene/Holocene Transition: Synthesis and Perspectives". Quaternary International 301: 23-33.

https://doi.org/10.1016/j.quaint.2012.05.017

Aceituno, F. J. y Uriarte, A. 2018: "Mobility and human dispersion during the peopling of Northwest South America between the Late Pleistocene and the Early Holocene". En R. Suárez y C. F. Ardelean (eds.): People and culture in Ice Age Americas: New dimensions in Paleoamerican Archaeology. University of Utah Press. Salt Lake City: 67-92.

Aguilella, G. 2011: “Cazadores-recolectores: más allá del territorio de explotación”. En V. Mayoral y S. Celestino (eds.): Tecnologías de Información Geográfica y Análisis Arqueológico del Territorio. Actas del $V$ Simposio Internacional de Arqueología de Mérida. Anejos AEspA LIX, CSIC. Mérida: 395-410.

Aubry, T.; Luís, L.; Mangado, J. y Matias, H. 2012: "We will be known by the tracks we leave behind: exotic lithic raw materials, mobility and social networking among the Coa Valley foragers (Portugal)". Journal of Anthropological Archaeology 31: 528-550. https://doi.org/10.1016/j.jaa.2012.05.003

Bell, T.; Wilson, A. y Wickham, A. 2002: "Tracking the Samnites: landscape and communications routes in the Sangro valley, Italy". American Journal of Archaeology 106 (2): 169-186. https://doi.org/10.2307/4126242

Bermúdez, J. 2006: "El análisis de las redes viarias en la antigüedad a partir de las posibilidades que ofrecen los SIG. Rutinas para el cálculo acumulado de vías óptimas con el programa Idrisi”. En I. Grau (ed.): La aplicación de los SIG en la arqueología del paisaje. Universidad de Alicante. Alicante: 91-97.

Bettinger, R. L. 1991: Hunter-gatherers: archaeological and evolutionary theory. Plenum Press. New York.

Binford, L. 1979: "Organization and formation processes: looking at curated technologies". Journal of Anthropological Research 35: 255-273. https://doi.org/10.1086/jar.35.3.3629902
Binford, L. 1980: "Wilow smoke and dog's tails: hunter-gatherer setlement systems and archaeological site formation". American Antiquity 45: 1-17. https://doi.org/10.2307/279653

Binford, L. 1987: "Researching ambiguity". En S. Kent (ed.): Method and theory for activity and research. Columbia University Press. New York: 449-512.

Binford, L. 1990: "Mobility, housing and environment: a comparative study". Journal of Anthropological Research (46): 119-152. https://doi.org/10.1086/jar.46.2.3630069

Binford, L. 2001: Constructing frames of reference: an analytical method for archaeological theory building using ethnographic and environment data. University of California Press. Berkeley y Los Angeles.

Botero, S. 2007: "Redescubriendo los caminos antiguos desde Colombia". Bulletin de l'Institut Français d'Etudes Andines 36 (3): 343-352. https://doi.org/10.4000/bifea.3505

Brantingham, J. 2006: "Measuring forager mobility". Current Anthropology 47 (3): 435-459. https://doi.org/10.1086/503062

Bruggencate, R. E.; Stup, J. P.; Milne, S. B.; Stenton, D. R.; Park, R. W. y Fayek, M. 2016: "A human-centered GIS approach to modeling mobility on southern Baffin Island, Nunavut, Canada". Journal of Field Archaeology 41 (6): 684-698. https://doi.org/10.1080/00934690.2016.1234897

Byrd, B. F.; Garrard, A. N. y Brandy, P. 2016: "Modeling foraging ranges and spatial organization of Late Pleistocene hunter-gatherers in the southern Levant - A least-cost GIS approach". Quaternary International 396: 62-78. https://doi.org/10.1016/j.quaint.2015.07.048

Cano, M. C. 2004: "Los primeros habitantes de las cuencas medias de los ríos Otún y Consota”. En C. E. López y M. C. Cano (eds.): Cambios ambientales en perspectiva histórica: Ecorregión del Eje Cafetero. Universidad Tecnológica de Pereira y Programa Ambiental GTZ, Cooperación Alemana al Desarrollo, Pereira, Colombia: 68-91.

Carballo, D. M. y Pluckhahn, T. 2007: “Transportation corridors and political evolution in highland Mesoamerica: Settlement analyses incorporating GIS for northern Tlaxcala, Mexico". Journal of Anthropological Archaeology 26 (4): 607-629. https://doi.org/10.1016/j.jaa.2007.05.001

Cardale, M. 2000: "Caminos al paisaje del pasado, reflexiones sobre los caminos precolombinos en Colombia". En L. Herrera y M. Cardale (eds.): Caminos precolombinos, las vías, los ingenieros y los viajeros. ICANH, Ministerio de Cultura. Bogotá: 43-86.

Cárdenas, D. y Politis, G. 2000: Territorio, movilidad, etnobotánica y manejo del bosque de los Nukak Orientales. En Colección de Estudios Antropológicos No 3. Universidad de los Andes. Bogotá.

Cashdan, E. 1983: "Territoriality among human foragers: ecological models and an application to four Bushman groups". Current Anthropology 24 (1): 47-66. https://doi.org/10.1086/202934

Conolly, J. y Lake, M. 2006: Geographical Information Systems in Archaeology. Cambridge University Press. New York.

Cooding, B. F. y Bird, D. W. 2016: "Behavioral ecology and the future of archaeological science". Journal of Archaeological Science 56: 9-20. https://doi.org/10.1016/j.jas.2015.02.027

Cortegoso, V.; Barberena, R.; Durán, V. y Lucero, G. 2016: “Geographic vectors of human mobility in the Andes (34-36 $\mathrm{S})$ : comparative analysis of 'minor' obsidian sources". Quaternary International 422: 81-92. https://doi.org/10.1016/j.quaint.2015.11.133

Cortés, L. 1978: "Los suelos en Colombia y su aptitud de uso". Colombia Geográfica 6 (1): 19-30.

Cowan, F. 1999: "Making sense of flake scatters: lithic technological strategies and mobility". American Antiquity 64 (4): 593-607. https://doi.org/10.2307/2694207

Criado, F. 1999: Del terreno al espacio: planteamiento y perspectivas para la arqueología del paisaje. CAPA 6. Universidad de Santiago de Compostela. Santiago de Compostela

Dickau, R.; Aceituno, F. J.; Loaiza, N.; López, C. E.; Cano, M.; Herrera, L.;... y Ranere, A. J. 2015: "Radiocarbon chronology of preceramic occupation in the Middle Cauca Valley, Colombia". Quaternary International 363: 43-54. https://doi.org/10.1016/j.quaint.2014.12.025

Dyson-Hudson, R. y Smith, E. 1978: "Human Territoriality". American Anthropologist 80 (1): 21-41.

https://doi.org/10.1525/aa.1978.80.1.02a00020 
Espinal, L.S. 1985: "Geografía ecológica del Departamento de Antioquia (zonas de vida formaciones vegetales) del Departamento de Antioquia". Revista Facultad Nacional de Agronomía 38 (1): 5-106.

Fábrega-Álvarez, P. 2006: "Moving without destination. A theoretical GIS-based determination of movement from a given origin". Archaeological Computing Newsletter 64: 7-11.

Fábrega-Álvarez, P. 2016: "Un alto en el camino. Notas acerca del uso de SIG en los análisis de movilidad en arqueología”. En M. C. Mínguez García y E. Capdevilla Montes (eds.): Manual de Tecnologías de la Información Geográfica aplicadas a la arqueología. Cursos de formación permanente para arqueólogos. Museo Arqueológico Regional del Madrid. Alcalá de Henares: 161-182.

Fábrega-Álvarez, P.; Fonte, J. y González, F. J. 2011: “Las sendas de la memoria. Sentido, espacio y reutilización de las estatuas-menhir en el noroeste de la Península Ibérica”. Trabajos de Prehistoria 68 (2): 313-330. https://doi.org/10.3989/tp.2011.11072

Fábrega-Álvarez, P. y Parcero-Oubiña, C. 2007: "Proposals for an archaeological analysis of pathways and movement". Archeologia $e$ Calcolatori 18: 121-140.

Fairén, S.; Cruz, M.; López-Romero, E. y Walid, S. 2006: "Las vías pecuarias como elementos arqueológicos". En I. Grau (ed.): La aplicación de los SIG en la arqueología del paisaje. Universidad de Alicante. Alicante: 55-68.

Field, J. S.; Petraglia, M. D. y Lahr, M. M. 2007: "The southern dispersal hypothesis and the South Asian archaeological record: examination of dispersal routes through GIS analysis". Journal of Anthropological Archaeology 26 (1): 88-108. https://doi.org/10.1016/j.jaa.2006.06.001

García-Moreno, A.; Fano, M. A. y Garate, D. 2012: “Integrating spatial analyses into foraging socities land use strategies. A case study from the Nalón River Basin (Asturias, North of Spain)". En G. Earl, T. Sly, A. Chrysanthi, P. Murrieta-Flores, C. Papadopoulos, I. Romanowska y D. Wheatley (eds.): Archaeology in the Digital Era. Papers from the $40^{\text {th }}$ Conference on Computer Applications and Quantitative Methods in Archaeology. Southampton, 26-30 March 2012. Amsterdam University Press. Amsterdam: 670-677.

Gorenflo, J. L. y Gale, N. 1990: "Mapping regional settlement in information space". Journal of Anthropological Archaeology 9: 240-274. https://doi.org/10.1016/0278-4165(90)90008-2

Grau, I. 2011: "Movimiento, circulación y caminos en el paisaje digital. La aplicación de los SIG en el estudio arqueológico de los desplazamientos humanos". En V. Mayoral y S. C. Pérez (eds.): Tecnologías de Información Geográfica y Análisis Arqueológico del Territorio. Actas del V Simposio Internacional de Arqueología de Mérida. Anejos AEspA LIX, CSIC. Mérida: 369-382.

Güimil-Fariña, A. y Parcero-Oubiña, C. 2015: “'Dotting the joins': a non-reconstructive use of Least Cost Paths to approach ancient roads. The case of the Roman roads in the NW Iberian Peninsula". Journal of Archaeological Science 54: 31-44. https://doi.org/10.1016/j.jas.2014.11.030

Herrera, L.; Moreno, M. C. y Peña, Ó. 2011: La historia muy antigua del municipio de Palestina (Caldas). Proyecto de Rescate y Monitoreo Arqueológico del Aeropuerto del Café. Centro de Museos, Universidad de Caldas, Asociación Aeropuerto del Café. Manizales, Colombia.

Howey, M. C. L. 2007: "Using multi-criteria cost surface analysis to explore past regional landscapes: a case study of ritual activity and social interaction in Michigan, AD 1200-1600". Journal of Archaeological Science 34 (11): 1830-1846. https://doi.org/10.1016/j.jas.2007.01.002

Jarvis, A.; Reuter, H. I.; Nelson, I. y Guevara, E. 2008: Hole-filled seamless SRTM data V4. International Centre for Tropical Agriculture (CIAT). http://srtm.csi.cgiar.org.

Kelly, R. L. 1992: "Mobility/sedentism: concepts, archaeological measures and effects". Annual Review of Anthropology 21: 43-66. https://doi.org/10.1146/annurev.anthro.21.1.43

Kelly, R. L. 1995: Foraging spectrum. Smithsonian Press. Washington.

Kelly, R. L. y Todd, L. C. 1988. "Coming into the country: early paleoindian hunting and mobility". American Antiquity 53 (2): 231-244. https://doi.org/10.2307/281017
Langebaek, K. 2001: "Reseña de caminos precolombinos, las vías, los ingenieros y los viajeros". Arqueología del Area Intermedia 3: 195-201.

Lee, R. y Daly, R. 1999: "Introduction: foragers and others". En R. Lee y R. Daly (eds.): The Cambridge Encyclopedia of hunters and gatherers. Cambridge University Press. Cambridge: 1-22.

Li, F.; Kuhn, S.; Chen, F. y Gao, X. 2016: "Raw material economies and mobility patterns in the Late Paleolithic at Shuidonggou locality 2, north China". Journal of Anthropological Archaeology 43: 83-93. https://doi.org/10.1016/j.jaa.2016.05.008

Llobera, M.; Fábrega-Álvarez, P. y Parcero-Oubiña, C. 2011: “Order in movement: a GIS approach to accessibility". Journal of Archaeological Science 38: 843-851. https://doi.org/10.1016/j.jas.2010.11.006

Loaiza, N. y Aceituno, F. J. 2015: "Reflexiones en torno al Arcaico colombiano". Revista Colombiana de Antropología 51 (2): 121-146.

Lurie, R. 1989: "Lithic technology and mobility strategies: the Koster site Middle Archaic". En R. Torrence (ed.): Time energy and stone tools. Cambridge University Press. Cambridge: 46-56.

Magnin, L.; Gobbo, D.; Gómez, J. C. y Ceraso, A. 2012: "GIS model of topographic accessibility to South America". En L. Miotti, M. Salemme, N. Flegenheimer y T. Goebel (eds.): Southbound: Late Pleistocene peopling of Latin America. Texas A\&M University College Station. Texas: 13-18.

Martínez i Rubio, T. y Martorell Briz, X. 2012: "La senda heredada: contribución al estudio de la red de caminos óptimos entre yacimientos de hábitat y de arte rupestre neolíticos en el Macizo del Caroig (Valencia, España)". Zephyrus (70): 69-84.

Melief, A. B. 1985: Late Quaternary Paleoecology of the Parque Nacional Natural los Nevados (Cordillera Central) and Sumapaz (Cordillera Oriental) Areas, Colombia. University of Amsterdam. Amsterdam.

Morgan, C. 2008: "Reconstructing prehistoric hunter-gatherer foraging radii: a case study from California's southern Sierra Nevada”. Journal of Archaeological Science 35 (2): 247-258. https://doi.org/10.1016/j.jas.2007.02.025

Morgan, C. 2009: "Climate change, uncertainty and prehistoric hunter-gatherer mobility". Journal of Anthropological Archaeology 28: 382-396. https://doi.org/10.1016/j.jaa.2009.07.004

Murrieta-Flores, P. 2012: "Understanding human movement through spatial technologies. The role of natural areas of transit in the Late Prehistory of South-western Iberia". Trabajos de Prehistoria 69 (1): 103-122. https://doi.org/10.3989/tp.2012.12082

Murrieta-Flores, P.; Wheatley, D. y García-Sanjuán, L. 2011: “Movilidad $\mathrm{y}$ vías de paso en los paisajes prehistóricos: megalitos y vías pecuarias en Almadén de la Plata (Sevilla, España)". En V. Mayoral y S. Celestino (eds.): Tecnologías de Información Geográfica y Análisis Arqueológico del Territorio. Actas del V Simposio Internacional de Arqueología de Mérida. Anejos AEspA LIX, CSIC. Mérida: 411-423.

Nelson, M. 1991: "The study of technological organization". En M. Schiffer (ed.): Archaeological Method and Theory 3, Academic Press. Nueva York: 57-100.

Odell, G. 2004: Lithic analysis. Springer Science. Nueva York.

Oyuela-Caicedo, A. 1990: "Las redes de caminos prehispánicos en la Sierra Nevada de Santa Marta". En S. Mora (ed.): Ingenierías prehispánicas. ICANH-FEN. Bogotá: 44-71.

Parcero-Oubiña, C.; Fábrega-Álvarez, P.; Güimil-Fariña, A.; Fonte, J. y Valdez, J. 2009: “Castros, caminos, rutas y ocupación del espacio. Modelización y análisis de las formas de movilidad asociadas a los asentamientos de la Edad del Hierro a través de herramientas SIG”. En F. Criado y A. Martínez (eds.): Arte rupestre, paleoambiente y paisaje. Miradas interdisciplinares sobre Campo Lameiro. TAPA 42. CSIC. Santiago de Compostela: 171-185.

Peterson, N. 1975: "Hunter-Gatherer territoriality: the perspective from Australia". American Anthropologist 77 (1): 53-68. https://doi.org/10.1525/aa.1975.77.1.02a00040

Politis, G. 1996: "Moving to produce: Nukak mobility and settlement patterns in Amazonia”. World Archaeology 27 (3): 492-511. https://doi.org/10.1080/00438243.1996.9980322

Politis, G. 2005: “Arqueología de carne y hueso”. Ciencia Hoy 16 (89): 44-50.

Trab. Prehist., 76, N. ${ }^{\circ}$ 2, julio-diciembre 2019, pp. 219-235, ISSN: 0082-5638

https://doi.org/10.3989/tp.2019.12234 
Rival, L. 1999: “The Huaorani”. En R. B. Lee y R. Daly (eds.): The Cambridge Encyclopedia of hunters and gatherers. Cambridge University Press. Cambridge: 101-109.

Rowley-Conwy, P. 2001: "Time, change and the archaeology of hunter-gatherers : how original is the 'Original afluent society'?". En Hunter-gatherers: an interdisciplinary perspective. Biosocial Society Symposium Series 13, Cambridge University Press. Cambridge: 39-72.

Sauvet, G. 2017: "The lifeword of hunter-gatherers and the concepts of territory". Quaternary International 503, Part B: 191-199. http://dx.doi.org/10.1016/j.quaint.2017.01.040.

Shoocongdej, R. 2000: "Forager mobility organization in seasonal tropical environments of western Thailand". World Archaeology 32 (1): 14-40. https://doi.org/10.1080/004382400409871

Shott, M. 1986: "Technological organization and settlement mobility: an ethnographic examination". Journal of Anthropological Research 42: 15-51. https://doi.org/10.1086/jar.42.1.3630378

Shott, M. 1996: "An exegesis of the curation concept". Journal of Anthropological Research 52 (3): 259-80. https://doi.org/10.1086/jar.52.3.3630085

Stiner, M. C. y Kuhn, S. L. 2016: "Are we missing the "sweet pot" between optimality theory and niche construction theory in archaeology?" Journal of Anthropological Archaceology 44: 177-184. https://doi.org/10.1016/j.jaa.2016.07.006

Thouret, J. C. 1983: "Presentación geológica y geomorfofuncional". En T. van der Hammen, A. Pérez y P. Pinto (eds.): Studies on tropical andean ecosystems (Estudios de ecosistemas tropandinos). La Cordillera Central colombiana transecto Parque de los Nevados (Introducción y datos iniciales) 1. Vaduz. J. Cramer. Berlin-Stuttgart: 48-55.
Torrence, R. 1983: "Time budgeting and hunter and gatherer technology". En G. Bailey (ed.): Hunter gatherer economy in prehistory: an european perspective. Cambridge University Press. Cambridge: 11-22.

Torrence, R. 1989: Time, energy and stone tools. Cambridge University Press. Cambridge.

Venkataraman, V. V.; Kraft, T. S.; Dominy, N. J. y Endicott, K. M. 2017: "Hunter-gatherer residential mobility and the marginal value of rainforest patches". Proceedings of the National Academy of Sciences 114 (12): 3097-3102. https://doi.org/10.1073/pnas.1617542114

Verhagen, P. 2013: "On the road to nowhere? Least cost paths accessibility and the predictive modelling perspective". En F. Contreras, M. Farjas y F. J. Melero (eds.): CAA2010, Fusion of Cultures. Proceedings of the $38^{\text {th }}$ Annual Conference on Computer Applications and Quantitative Methods in Archaeology, Granada, Spain, April 2010. British Archaeological Reports, International Series 2494, Archaeopress. Oxford: 383-389.

Villaverde, V.; Martínez, T.; Guillem, P. M.; Martínez, R. y Martínez J. A. 2016: "Arte rupestre y hábitat en la prehistoria del Riu de les Coves. Aproximación a la cronología del Arte Levantino a través de la red de caminos óptimos". En Del neolitic a l'edat del bronze en el Mediterrani occidental. Estudis en homenatge a Bernat Martí Oliver. Serie Trabajos Varios 119, SIP. Valencia: 501-520.

Wheatley, D. y Gillings, M. 2002: Spatial technology and archaeology. The archaeological applications of GIS. Taylor \& Francis. New York.

White, D. A. y Barber, S. B. 2012: "Geospatial modeling of pedestrian transportation networks: a case study from precolumbian Oaxaca, Mexico". Journal of Archaeological Science 39 (8): 2684-2696. https://doi.org/10.1016/j.jas.2012.04.017 